\title{
Contribution of changes in atmospheric circulation patterns to extreme temperature trends
}

Daniel E. Horton ${ }^{1,2 *}$, Nathaniel C. Johnson ${ }^{3,4,5}$, Deepti Singh ${ }^{1}$, Daniel L. Swain ${ }^{1}$, Bala Rajaratnam $^{1,2,6}$, and Noah S. Diffenbaugh ${ }^{1,2}$

${ }^{1}$ Department of Earth System Science, Stanford University, Stanford, CA 94305, USA

${ }^{2}$ Woods Institute for the Environment, Stanford University, Stanford, CA 94305, USA

${ }^{3}$ International Pacific Research Center, University of Hawaii at Manoa, Honolulu, HI 96822, USA

${ }^{4}$ Scripps Institution of Oceanography, University of California San Diego, La Jolla, CA 92093, USA

${ }^{5}$ Cooperative Institute for Climate Science, Princeton University, Princeton, NJ 08540, USA

${ }^{6}$ Department of Statistics, Stanford University, Stanford, CA, 94305, USA

*Corresponding author. Email: danethan@stanford.edu 


\section{Contribution of changes in atmospheric circulation patterns to extreme temperature trends}

Surface weather conditions are closely governed by the large-scale circulation of the atmosphere. Recent increases in the occurrence of some extreme weather phenomena ${ }^{1,2}$ have led to multiple mechanistic hypotheses linking changes in atmospheric circulation to increasing extreme event probability ${ }^{3-5}$. However, observed evidence of long-term change in atmospheric circulation remains inconclusive $\mathrm{e}^{6-8}$. Here we identify statistically significant trends in the occurrence of mid-atmospheric circulation patterns, which partially explain observed trends in surface temperature extremes over seven mid-latitude regions of the Northern Hemisphere. Utilizing self-organizing map (SOM) cluster analysis ${ }^{9-12}$, we detect robust pattern trends in a subset of these regions during both the satellite observation era (1979-2013) and the recent period of rapid Arctic sea ice decline (1990-2013). Particularly substantial influences include the contribution of increasing trends in anticyclonic circulations to summer/autumn hot extremes over portions of Eurasia and North America, and the contribution of increasing trends in northerly flow to winter cold extremes over central Asia. Our results indicate that although a substantial portion of the observed change in extreme temperature occurrence has resulted from regional- and global-scale thermodynamic changes, the risk of extreme temperatures over some regions has also been altered by recent changes in the frequency, persistence, and/or maximum duration of regional circulation patterns.

Although most land regions show robust warming over the past century ${ }^{13}$, the pattern of change has not been spatially uniform ${ }^{14}$. This heterogeneity results from regional differences in the response of the climate system to increasing radiative forcing, and from the background noise of climate variability. Together, these factors substantially increase the challenge of climate change detection, attribution, and projection at regional and local scales ${ }^{14,16}$.

The spatial pattern of changes in extreme weather events has generated arguments that global warming has caused dynamic and/or thermodynamic changes that have differentially altered extreme event probabilities ${ }^{1,17}$. Thermodynamic arguments are well 
30 understood and observed. For example, the accumulation of heat in the atmosphere has

31 resulted in upward trends in hot extremes, downward trends in the majority of cold 32 extremes, and more intense hydroclimatic events ${ }^{1,2}$. Dynamic arguments have greater 33 uncertainties ${ }^{15-19}$. Changes in the large-scale atmospheric circulation - for instance, an 34 increase in the occurrence or persistence of high-amplitude wave patterns - could alter 35 the likelihood of extreme events ${ }^{20}$. Recent extremes in the Northern Hemisphere mid36 latitudes"1,2,17 have motivated hypotheses of a dynamic linkage between "Arctic 37 Amplification", altered atmospheric circulation patterns, and changes in the probability of 38 mid-latitude extremes ${ }^{\text {e.g.,3-5,17 }}$. Despite divergent views on the causal direction of this 39 linkage ${ }^{17}$, altered atmospheric dynamics are consistently invoked. Although trends in 40 mean-seasonal mid-atmospheric geopotential height anomalies have been identified (Fig. 412.36 ref. 21; Fig. 1), evidence of changes in the occurrence of sub-seasonal atmospheric 42 patterns remains equivocal, as does their contribution to extreme event probabilities ${ }^{6-8}$.

43 Previous efforts to detect trends in atmospheric circulation may have been 44 hampered by narrowly-defined, spatially-sensitive, and/or non-standardized metrics ${ }^{3,6-8,17}$. 45 We therefore employ a large-scale spatial characterization approach - Self-Organizing 46 Map ("SOM") cluster analysis - to track the occurrence of highly generalized mid47 atmospheric circulation patterns. We use $500 \mathrm{hPa}$ geopotential height anomaly fields to 48 describe daily circulation, and group each day's pattern into one of a predefined number 49 of SOM clusters based on a measure of pattern similarity ${ }^{9-12}$ (Methods). The number of 50 clusters is largely dependent on the degree of specificity/generality required to test a 51 particular hypothesis ${ }^{9-12}$. To facilitate generalized large-scale mid-atmospheric 52 classification, we utilize four clusters per domain. Using three reanalyses (NCAR/NCEP$53 \mathrm{R} 1$, NCEP-DOE-R2, and ERA-Interim), we calculate linear trends $\left[\mathrm{yr}^{-1}\right.$ ] in the time54 series of annual values of ( $i$ ) the total number of days in each season on which each SOM 55 pattern occurs ("occurrence"; $\left[\mathrm{d} \bullet \mathrm{yr}^{-1}\right]$ ), (ii) the mean length of consecutive occurrence 56 ("persistence"; [d•event $\left.{ }^{-1}\right]$ ), and (iii) the longest consecutive occurrence ("maximum 57 duration"; $\left.\left[\mathrm{d} \bullet e^{-1} \mathrm{ent}^{-1}\right]\right)$. We consider trends in each metric robust if matching circulation 58 patterns from all three reanalyses have statistically significant trends of the same sign. 59 We assess the robustness of trends for seven mid-latitude regions (Fig. 2a), over both the 60 satellite era (1979-2013; "sat-era”) and the rapidly-diminishing Arctic sea ice era ${ }^{22}$ 
61 (1990-2013; “ice-era"). We report circulation patterns that pass these robustness criteria,

62 but also discuss results in the context of $(i)$ comprehensive multiple hypothesis testing,

63 (ii) removal of the assumption of linear time-series relationships, (iii) use of fewer/more 64 clusters, and (iv) addition of atmospheric thermal dilation controls (Methods; ED_Table 1 65 and ED_Figs. 1-3).

66 Of the 112 total circulation patterns analyzed in each period (Methods), the three 67 reanalyses exhibit statistically significant trends in pattern occurrence for a total of 17 , 68 16, and 16 patterns in the sat-era, and 15, 13, and 14 patterns in the ice-era (ED_Table 69 1a). Of these significant occurrence trends, 12 sat-era and 10 ice-era patterns are robustly 70 significant across all three reanalyses (Table 1). The majority of robust sat-era trends 71 occur in summer and autumn, while robust ice-era trends are more evenly distributed 72 over summer, autumn, and winter. These patterns are diverse, and include anticyclonic, 73 cyclonic, and "dipole" circulations (ED_Figs. 4-5). Patterns with robust trends in both 74 sat- and ice-eras are limited to summer and autumn over western Asia and eastern North 75 America.

76 While the number of significant trends in pattern persistence varies from 5 to 10 77 across the individual reanalyses (ED_Table 1a), only three robust pattern persistence 78 trends are identified in each period (Table 1). Robust maximum duration trends are more 79 prevalent, including five in the sat-era and six in the ice-era. These are predominantly 80 associated with summer anticyclonic patterns, although the maximum duration of central 81 Asia winter troughing events demonstrates a robust ice-era increase (ED_Figs. 4-5). In 82 regions with robust trends in multiple patterns, those patterns are generally 83 complimentary. For example, in summer over eastern North America, robustly increasing 84 sat-era trends in anticyclonic patterns co-occur with robustly decreasing trends in 85 cyclonic patterns.

86 To what extent have mid-atmospheric circulation trends influenced the likelihood 87 of temperature extremes? For each period, we compute area-weighted trends in the 88 seasonal occurrence of temperature extremes for all days, and for those days associated 89 with each SOM pattern (e.g., Fig. 2a, j-m; Methods). The three reanalyses generally agree 90 on the direction of all-days trends: consistent with enhanced radiative forcing and global 
91 warming, most regions and seasons show positive trends in hot occurrence, and negative 92 trends in cold occurrence (Table 1).

93 Hot extremes are projected to increase due to the dynamic and thermodynamic 94 effects of global warming ${ }^{1,23}$. Consistent with other assessments ${ }^{1,2}$, we find substantial 95 increases in extreme heat occurrence over the mid-latitudes (ED_Figs. 6-7). For instance, 96 the regional-mean occurrence of summer hot days over Europe, western Asia and eastern 97 North America has increased 0.10, 0.16, and $0.13 \mathrm{~d} \cdot \mathrm{yr}^{-1} \cdot \mathrm{yr}^{-1}$, respectively, over the sat98 era (Fig. 2a, ED_Table 2a-c). By definition, one would expect (on average) $4.55^{\text {th }} / 95^{\text {th }}$ 99 percentile events per 3-month season, meaning that an increase of $0.10 \mathrm{~d} \bullet \mathrm{yr}^{-1} \cdot \mathrm{yr}^{-1}$ 100 accumulated over the course of the sat-era (35 years) yields an additional $\sim 3.5 \mathrm{~d} \bullet \mathrm{yr}^{-1}$, an $101 \sim 75 \%$ increase.

102 Heatwaves, similar to those which occurred in western Russia in 2010 and Europe 103 in 2003, develop when persistent anticyclonic patterns, often referred to as "atmospheric 104 blocking", initiate a cascade of self-reinforcing, heat-accumulating physical 105 processes $^{24,25}$. In addition to the increasing trends in extreme heat occurrence, robust 106 positive trends in the occurrence, persistence, and maximum duration of sat-era summer 107 mid-atmospheric anticyclonic patterns are detected over Europe (Fig. 2c, g), western Asia 108 (Fig. 3a,e), and eastern North America (ED_Fig. 4c). Robust positive trends in the 109 occurrence of sat-era anticyclonic patterns are also detected - along with increasing heat 110 extremes - in autumn over eastern North America (Fig. 3c,g), eastern Asia (Fig. 3d,h) 111 and central North America, and in spring over Europe (ED_Fig. 1).

112 Increases in hot extremes may result from dynamic changes (namely greater 113 occurrence and persistence of anticyclonic patterns), and/or from the thermodynamic 114 effects of global warming (reflected in the increased intensity of extreme temperature 115 when anticyclonic patterns occur). Over Europe, the summer occurrence of circulations 116 similar to dipole patterns with ridging over the eastern half of the domain (Fig. 2c) 117 increased $0.45 \mathrm{~d} \cdot \mathrm{yr}^{-1} \cdot \mathrm{yr}^{-1}$ over the sat-era, while the persistence and maximum duration 118 increased 0.05 and $0.19 \mathrm{~d} \cdot$ event $^{-1} \cdot \mathrm{yr}^{-1}$, respectively (Fig. $2 \mathrm{~g}$ ). The trend in the frequency 119 of hot events coincident with this pattern (0.06 d• $\mathrm{yr}^{-1} \cdot \mathrm{yr}^{-1}$; Fig. 2k, ED_Table 2a) 120 accounts for $62 \%$ of the total trend in hot extremes over Europe $\left(0.10 \mathrm{~d} \bullet \mathrm{yr}^{-1} \cdot \mathrm{yr}^{-1}\right.$; Fig. 2a). 121 In addition, the number of hot extremes per pattern occurrence has increased for all four 
122 patterns (Fig. 2n-q, ED_Table 2a). Under the assumption of pattern stationarity, we

123 perform a quantitative partitioning of the dynamic and thermodynamic contributions to 124 extreme temperature trends ${ }^{10}$ (Methods). This partitioning reveals that $57 \%$ of the trend 125 in hot extremes associated with this pattern is driven by thermodynamic influences, while $12644 \%$ is due to the dynamic influence of increased pattern occurrence (ED_Table 2a). 127 Together, these results suggest that the observed increase in extreme summer heat over 128 Europe is attributable to both increasing frequency of blocking circulations and changes 129 in the surface energy balance. Similar results are found in other regions that exhibit 130 robust upward trends in anticyclonic patterns (Fig. 3, ED_Table 2).

131 Global warming is also generally expected to decrease the frequency of cold 132 extremes ${ }^{1}$. In autumn over eastern Asia, the occurrence of sat-era cold extremes 133 decreased $0.08 \mathrm{~d} \cdot \mathrm{yr}^{-1} \cdot \mathrm{yr}^{-1}$, indicating a reduction of $\sim 60 \%$ over the 35 year period

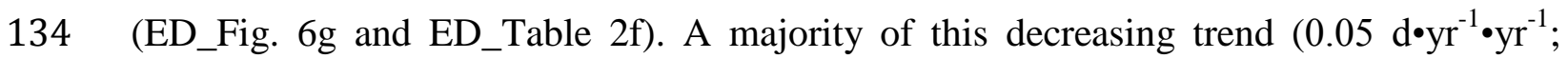
135 ED_Table 2f) is attributable to changes associated with one pattern type: cyclonic 136 circulations capable of advecting cold air equatorward (Fig. 3d). Less frequent 137 occurrence of cyclonic patterns (Fig. 3h), in conjunction with less intense cold 138 temperature anomalies when cyclonic patterns occur (Fig. 3p), drive 63\% of the overall

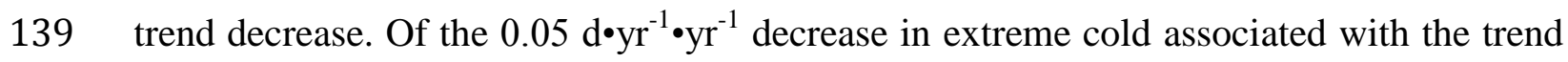
140 in cyclonic patterns, partitioning indicates 58\% dynamic and 35\% thermodynamic 141 contributions (ED_Table 2).

142 In contrast to this expected extreme cold decrease, winter cold extremes over

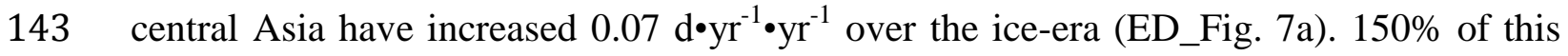
144 trend $\left(0.10 \mathrm{~d} \bullet \mathrm{yr}^{-1} \cdot \mathrm{yr}^{-1}\right.$; Fig. $\left.3 \mathrm{j}\right)$ occurred when mid-atmospheric circulation was similar to 145 a pattern of troughing in the south and east, and ridging in the northwest (Fig. 3b). (Trend 146 percentages exceeding $100 \%$ indicate that other circulation patterns provide negative 147 contributions (ED_Table 2).) Occurrence and persistence of this dipole pattern robustly 148 increased $\left(1.0 \mathrm{~d} \cdot \mathrm{yr}^{-1} \cdot \mathrm{yr}^{-1}\right.$ and $0.12 \mathrm{~d} \cdot e^{2} \operatorname{cnt}^{-1} \cdot \mathrm{yr}^{-1}$, Fig. 3f $)$ at the expense of all other 149 circulations (ED_Table 2d). Partitioning indicates that 75\% of the extreme cold trend 150 associated with this pattern is due to the dynamic influence of increased pattern 151 occurrence, with 18\% linked to thermodynamic influences (ED_Table 2d). 
152 Substantial dynamic contributions to the overall trend in cold extremes could be 153 expected given that circulations that support the equatorward advection of Arctic air will 154 bring anomalously cold temperatures to lower-latitude locales ${ }^{20}$. Increased occurrence of 155 such patterns has previously been observed, and linked to reduced regional sea-ice and 156 decreased baroclinicity over the Barents-Kara Seas ${ }^{4,17,26-28}$. Positive thermodynamic 157 contributions to the extreme cold trend indicate processes that are in opposition to the 158 direct warming effects of enhanced radiative forcing. For example, positive 159 thermodynamic contributions from 3 of the 4 winter patterns over central Asia 160 (ED_Table 2d) suggest that these contributions are largely independent of atmospheric 161 circulation, and therefore potentially related to surface processes such as increased snow 162 cover and enhanced diabatic cooling ${ }^{4,28}$.

163 The circulation trends detected here cannot as yet be attributed to anthropogenic 164 or natural causes, nor can they be projected to continue into the future. Attribution and 165 projection will require an increased understanding of the causes of the circulation trends, 166 including the ability to identify the signal of an anthropogenically forced trend from the 167 noise of internal decadal-scale climate variability ${ }^{16,29}$. However, our quantitative 168 partitioning, in conjunction with targeted climate model simulations ${ }^{16,29,30}$, offers the 169 potential to fingerprint dynamic and thermodynamic climate influences in isolation, 170 which in turn may facilitate attribution of the observed trends, and projection of future 171 trends. We hypothesize that the main assumption of our quantitative partitioning - pattern 172 stationarity - is justified given the expectation that circulation responses to enhanced 173 radiative forcing are likely to reinforce preexisting modes of natural variability ${ }^{15,16}$. A 174 related assumption is that the reanalyses act as reasonable proxies for the state of the 175 three-dimensional atmosphere through time. Given uncertainties in the data assimilation 176 and numerical modeling that underpin atmospheric reanalysis, we have restricted our 177 identification criteria to those trends that are statistically significant in all three 178 reanalyses.

179 Our approach finds robust trends in mid-atmospheric circulation patterns over 180 some regions, and suggests that both dynamic and thermodynamic effects have 181 contributed to observed changes in temperature extremes over the past 35 years. 182 Although thermodynamic influences have largely dominated these changes, dynamic 
183 influences have been critical in some regions and seasons. Long-term projections of 184 future dynamic contributions are challenging given the substantial underlying decadal185 scale variability, as well as the uncertain impact of anthropogenic forcing on mid-latitude

186 circulation ${ }^{15,16}$. However, given our finding that many patterns have exhibited increasing

187 (decreasing) intensity of extreme hot (cold) events, and that those trends are coincident 188 with a nearly categorical increase in thermodynamic forcing, the observed trends of 189 increasing hot extremes and decreasing cold extremes could be expected to continue in 190 the coming decades, should greenhouse gases continue to accumulate in the atmosphere.

\section{References}

194 1. Field, C. B. et al. (eds) Managing the Risks of Extreme Events and Disasters to 195 Advance Climate Change Adaptation (Cambridge Univ. Press, 2012).

2. Donat, M. G. et al. Updated analyses of temperature and precipitation extreme indices

198 since the beginning of the twentieth century: The HadEX2 dataset. J. Geophys. Res. 118, 199 2098-2118 (2013).

201 3. Francis, J. A. \& Vavrus, S. J. Evidence linking Arctic amplification to extreme weather 202 in mid-latitudes. Geophys. Res. Lett. 39, L06801 (2012).

204 4. Liu, J., Curry, J. A., Wang, H., Song, M. \& Horton, R. M. Impact of declining Arctic 205 sea ice on winter snowfall. Proc. Natl Acad. Sci. USA 109, $4074-4079$ (2012).

207 5. Petoukhov, V., Rahmstorf, S., Petri, S. \& Schellnhuber, H. J. Quasiresonant

208 amplification of planetary waves and recent Northern Hemisphere weather extremes.

209 Proc. Natl Acad. Sci. USA 110, 5336-5341 (2013).

211 6. Screen, J. A. \& Simmonds, I. Caution needed when linking weather extremes to

212 amplified planetary waves. Proc. Natl Acad. Sci. USA 110, E2327 (2013). 
214 7. Barnes, E. A., Dunn-Sigouin, E., Masato, G. \& Woolings, T. Exploring recent trends in 215 Northern Hemisphere blocking. Geophys. Res. Lett. 41, 638-644 (2014).

217 8. Screen, J. \& Simmonds, I. Exploring links between Arctic amplification and mid-

218 latitude weather. Geophys. Res. Lett. 40, 959-964 (2013).

9. Kohonen, T. Self-Organizing Maps p. 501, (Springer, 2001)

10. Cassano, J. J., Uotila, P., Lynch, A. H. \& Cassano, E. N. Predicted changes in synoptic forcing of net precipitation in large Arctic river basins during the $21^{\text {st }}$ century. $J$.

224 Geophys. Res. 112, G04S49 (2007).

225

226 11. Johnson, N. C., Feldstein, S. B. \& Tremblay, B. The continuum of Northern

227 Hemisphere teleconnection patterns and a description of the NAO shift with the use of

228 self-organizing maps. J. Climate 21, 6354-6371 (2008).

12. Lee, S. \& Feldstein, S. B. Detecting ozone- and greenhouse gas-driven wind trends 232 with observational data. Science 339, 563-567 (2013).

13. Diffenbaugh, N. S. et al. in Climate Change 2014: Impacts, Adaptation, and 235 Vulnerability (eds. Field, C. B. et al.) 137-141 (IPCC, Cambridge Univ. Press, 2014).

14. Field C. B. et al. in Climate Change 2014: Impacts, Adaptation, and Vulnerability 238 (eds. Field, C. B. et al.) 1-32 (IPCC, Cambridge Univ. Press, 2014).

240 projections. Nature Geosci. 7, 703-708 (2014).

242 16. Deser, C., Phillips, A. S., Alexander, M. A. \& Smoliak, B. V. Projecting North 243 American climate over the next 50 years: uncertainty due to internal variability. $J$. 244 Clim. 27, 2271-2296 (2014). 
246 17. Cohen J. et al. Recent Arctic amplification and extreme mid-latitude weather. Nature

247 Geosci. 7, 627-637 (2014).

249 18. Palmer, T. Record-breaking winters and global climate change. Science 344, 803-804 250 (2014).

252 19. Wallace, J. M., Held, I. M., Thompson, D. W. J., Trenberth, K. E. \& Walsh, J. E.

253 Global warming and winter weather. Science 343, 729-730 (2014).

255 20. Screen, J. A. Arctic amplification decreases temperature variance in northern mid- to 256 high-latitudes. Nature Clim. Change 4, 577-582 (2014).

258 21. Hartmann, D. L. et al. in Climate Change 2013: The Physical Science Basis (eds.

259 Stocker, T. F. et al.) 159-254 (IPCC, Cambridge Univ. Press, 2013).

261 22. Simmonds, I. Comparing and contrasting the behaviour of Arctic and Antarctic sea

262 ice over the 35-year period 1979-2013. Ann. Glaciol. 56, 18-28 (2015).

264 23. Diffenbaugh N.S. \& Ashfaq, M. Intensification of hot extremes in the United States.

265 Geophys. Res. Lett. 37, L15701 (2010).

267 24. Miralles, D. G., Teuling, A. J., van Heerwaarden, C. C. \& Vila-Guerau de Arellano, J.

268 Mega-heatwave temperatures due to combined soil desiccation and atmospheric heat

269 accumulation. Nature Geosci. 7, 345-349 (2014).

271 25. Diffenbaugh, N. S., Pal, J. S., Trapp, R. J. \& Giorgi, F. Fine-scale processes regulate 272 the response of extreme events to global climate change. Proc. Natl Acad. Sci. USA 102, 273 15774-15778 (2005). 
275 26. Inoue, J., Hori, M. E. \& Takaya, K. The role of Barents Sea ice in wintertime cyclone 276 tracks emergence of a warm-Artic cold-Siberian Anomaly. J. Climate 25, 2561-2568

277 (2012).

278

279 27. Mori, M., Watanabe, M., Shiogama, H., Inoue, J. \& Kimoto, M. Robust Arctic sea-ice 280 influence of the frequent Eurasian cold winters in past decades. Nature Geosci. 7, 869281873 (2014).

283 28. Cohen, J., Furtado, J., Barlow, J. M., Alexeev, V. \& Cherry, J. Arctic warming, 284 increasing fall snow cover and widespread boreal winter cooling. Environ. Res. Lett. 7, 285014007 (2012).

29. Screen, J.A., Deser, C., Simmonds, I. \& Tomas, R. Atmospheric impacts of Arctic sea-ice loss, 1979-2009: Separating forced change from atmospheric internal variability. Climate Dyn. 43, 333-344 (2014).

30. Screen, J.A., Simmonds, I., Deser, C. \& Tomas, R. The atmospheric response to three

292 decades of observed Arctic sea ice loss. J. Climate 26, 1230-1248 (2013).

\section{Acknowledgements}

Work by D.E.H., D.S., D.L.S., and N.S.D. was supported by NSF CAREER 296 Award 0955283, DOE Integrated Assessment Research Program Grant No. DE297 SC005171DE-SC005171, and a G.J. Lieberman Fellowship to D.S. Contributions from 298 N.C.J. were supported by NOAA's Climate Program Office's Modeling, Analysis, 299 Predictions, and Projections program award \#NA14OAR4310189. B.R. acknowledges 300 support from the US Air Force Office of Scientific Research (FA9550-13-1-0043), the

301 US National Science Foundation (DMS-0906392, DMS-CMG-1025465, AGS-1003823, 302 DMS-1106642, and DMS-CAREER-1352656), the Defense Advanced Research Projects 303 Agency (DARPA YFA N66001-111-4131), and the UPS Foundation (SMC-DBNKY).

304 We thank J. Cattiaux, D. Touma, and J.S. Mankin for discussions that improved the 305 manuscript. Computational resources for data processing and analysis were provided by 
306 the Center for Computational Earth and Environmental Science in the School of Earth,

307 Energy, and Environmental Sciences at Stanford University.

308

\section{Author Contributions}

D.E.H. conceived the study. D.E.H., N.C.J., D.S., D.L.S., and N.S.D. designed the

311 analysis and co-wrote the manuscript. D.E.H., N.C.J., and D.S. provided analysis tools.

312 D.E.H. performed the analysis. B.R. provided and described the multiple hypothesis

313 testing and transformation analysis.

315 Competing Financial Interests

316 The Authors declare no competing financial interests.

318 Corresponding Author

319 Daniel E. Horton, danethan@stanford.edu

Figure \& Table Legends

Table 1 | Trends in surface temperature extremes and atmospheric circulation

324 patterns. Trends are calculated for each NH season (DJF-winter, MAM-spring, JJA325 summer, SON-autumn) for two periods, 1979-2013 (sat-era) and 1990-2013 (ice-era).

326 Regional domains (see Fig. 2a) in which one or more of the four SOM circulation 327 patterns demonstrate robust trends in mid-atmospheric circulation pattern occurrence $(O)$, 328 persistence $(\mathrm{P})$, or maximum duration $(\mathrm{M})$ are shown in green (ED_Figs 1-2). Positive 329 (+) and negative (-) symbols are displayed when all three reanalyses show statistically 330 significant trends in a particular circulation pattern, and agree on the sign of those trends. 331 Multiple symbols within a box indicate multiple robust pattern trends. White boxes

332 without symbols indicate no statistically significant trends and/or reanalysis disagreement 333 (see Methods). Regional domains with positive and/or negative trends in cold (05) and/or 334 hot (95) extremes receive (+) or (-) symbols when the three reanalyses agree on the sign 335 of the area-weighted trend. Red and blue boxes indicate that the extreme temperature 
336 trend results in warming and cooling, respectively, while gray boxes indicate reanalysis 337 disagreement.

339 Figure 1 | Trends in mid-atmospheric geopotential height anomalies. Northern

340 Hemisphere polar projections of 1979-2013 seasonal trends $\left[\mathrm{m}^{\bullet} \mathrm{yr}^{-1}\right]$ in $500 \mathrm{hPa}$ 341 geopotential height anomalies. The time-series of seasonal mean anomalies is calculated 342 from the daily anomalies generated by subtracting the seasonal cycle from each grid cell.

343 Trends are computed for a, winter (DJF), b, spring (MAM), c, summer (JJA), and d, 344 autumn (SON) seasons. Geopotential height fields are sourced from NCEP-DOE-R2.

346 Figure 2 | Trends in circulation patterns and hot extremes over Europe. a, 19793472013 trends in summer hot extreme occurrences for all regional domains based on 2348 meter maximum/minimum temperatures from NCEP-DOE-R2. b-e, SOM-derived mid349 atmospheric circulation patterns (500 hPa geopotential height anomalies) over Europe. 350 White-boxed values show pattern frequencies (\%) in the top left, and SOM node numbers 351 in the top right. f-i, Time-series of SOM circulation pattern occurrence (black; $\left[\mathrm{d} \bullet \mathrm{yr}^{-1}\right]$ ), 352 persistence (blue; $\left[\mathrm{d} \bullet e^{-} e^{-1}{ }^{-1}\right]$ ), and maximum duration (red; [d•event $\left.{ }^{-1}\right]$ ). The slope of the 353 trend line $\left[\mathrm{yr}^{-1}\right]$ and $\mathrm{p}$-values (in parentheses) are color coded, with the values from 19793542013 (solid trend line) displayed above those from 1990-2013 (dashed trend line). j-m, 355 Spatially rendered trends in hot extreme occurrences for days that correspond to each 356 SOM circulation pattern. n-q, Time-series of the area-weighted mean of hot extremes per 357 pattern occurrence, referred to throughout the text as a measure of the intensity of 358 temperature extremes associated with each pattern. Statistically significant trends (5\% 359 significance level) are shown by stippling in the mapped panels and by bold fonts in the 360 scatter plots.

362 Figure 3 | Circulation pattern and thermal extreme trends for selected regions.

363 Trends in thermal extreme occurrences for selected regions and seasons based on 2-meter 364 maximum/minimum temperatures from NCEP-DOE-R2. a-d, SOM-derived mid365 atmospheric circulation patterns (500 hPa geopotential height anomalies) over western 366 Asia in summer (a), central Asia in winter (b), eastern North America in autumn (c), and 
367 eastern Asia in autumn (d). White-boxed values show pattern frequencies (\%) in the top 368 left, and SOM node numbers in the top right. In contrast to Fig. 2, just one of the four 369 SOM circulation patterns is displayed from each region. e-h, Time-series of SOM

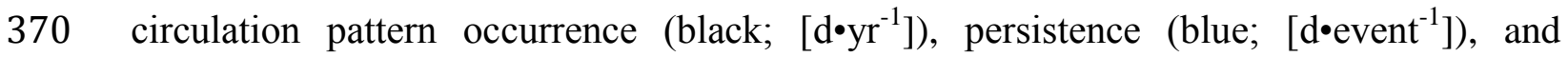

371 maximum duration (red; $\left.\left[\mathrm{d} \bullet \mathrm{event}^{-1}\right]\right)$. The slope of the trend line $\left[\mathrm{yr}^{-1}\right]$ and $\mathrm{p}$-values (in 372 parentheses) are color coded, with the values from 1979-2013 (solid trend line) displayed 373 above those from 1990-2013 (dashed trend line). i-l, Spatially rendered trends in thermal 374 extreme occurrences for days that correspond to each SOM circulation pattern. m-p, 375 Time-series of the area-weighted mean of temperature extremes per pattern occurrence, 376 referred to throughout the text as a measure of the intensity of temperature extremes 377 associated with each pattern. Statistically significant trends (5\% significance level) are 378 shown by stippling in the mapped panels and by bold fonts in the scatter plots. Refer to 379 Extended Data Figs. 6 and 7 for sat-era and ice-era trends in temperature extremes over 380 the regional domains.

382 Methods

383 Categorization of Circulation Patterns

384 We use SOM cluster analysis ${ }^{9-12}$ to categorize large-scale circulation patterns over 385 seven Northern Hemisphere domains ${ }^{31}$ using daily $500 \mathrm{hPa}$ geopotential height anomaly 386 fields from the NCAR/NCEP-R1 (32), NCEP-DOE-R2 (33), and ECMWF ERA-Interim 387 (34) reanalyses. Daily anomalies are calculated by subtracting the seasonal cycle 388 (calendar-day mean) from each grid cell. Reanalyses are analyzed individually to 389 maintain their physical consistency, and to facilitate their intercomparison. The SOMs' 390 unsupervised learning algorithm requires neither a priori knowledge of which types of 391 circulation patterns might be detected, nor the specific geographic regions in which they 392 might occur. Geopotential height anomaly fields from each day are assigned to one of a 393 pre-defined number of nodes, according to pattern similarity. The final SOM patterns are 394 obtained by minimizing the Euclidian distance between iteratively updated nodes and 395 their matching daily geopotential height anomaly fields ${ }^{11}$. Each SOM pattern can 396 therefore be viewed as a representative composite of relatively similar circulation 397 patterns. 
Due to global-scale warming, trends in geopotential height anomalies record both altered atmospheric circulation patterns and the thermal expansion of the troposphere. To 400 isolate the signal of circulation pattern change, previous clustering analyses have 401 assumed uniform thermal dilation and removed either the domain average ${ }^{35}$ or domain 402 average linear trend ${ }^{36}$ from the daily-scale anomalies. In our analysis, we find that 19794032013 trends in Northern Hemisphere geopotential height anomalies are non-uniform in 404 both magnitude and sign (Fig. 1), and demonstrate substantial seasonal, regional, and 405 latitudinal differences (ED_Fig. 3e). These findings suggest that for the relatively short 406 period of our analysis, an assumption of uniform thermal dilation is inappropriate. 407 Moreover, the strong spatial heterogeneity indicates the importance of large-scale 408 dynamics in the regional geopotential height trends, and so the removal of local 409 geopotential height trends would conflate dynamic changes with thermal dilation. 410 Therefore, in the main text we present results and conclusions based on raw geopotential 411 height data. However, despite the lack of uniform expansion, we have conducted an 412 analysis that attempts to account for the effects of thermal dilation. SOM analyses are 413 performed on geopotential heights that have been detrended by removing the seasonal 414 mean hemispheric trend (ED_Fig. 3e) from each grid cell. Results from this analysis 415 indicate that the magnitude, significance, and sign of circulation trends are sensitive to 416 the method of controlling for thermal expansion (ED_Fig. 3f-j). Despite this sensitivity, 417 the conclusions presented in the main text are supported, in that both raw and detrended 418 analyses generally suggest trends of similar magnitude, sign, and significance.

419 Based on domain-wide pattern correlations between daily height field anomalies 420 and different SOM node counts ${ }^{12}$ (ED_Fig. 8), we divide circulation patterns over each 421 domain into four SOM nodes (e.g., Fig. 2b-e). To determine a suitable number of nodes, 422 a suite of different node counts were analyzed. We found that four nodes were 423 sufficiently great in number to capture a diversity of highly generalized circulation 424 patterns, but sufficiently few to facilitate convenient presentation and - critically - to 425 prevent overly similar SOM patterns ${ }^{12}$. To test the sensitivity of our results to the number 426 of nodes, we present 2-, 4-, 8-, and 16-node SOMs for the summer season over the 427 European domain (ED_Figs. 1-2). Based on these analyses, it is apparent that a 2-node 428 SOM is insufficient to capture the diversity of circulation patterns that are found in the 
429 reanalysis data (ED_Fig. 1a), whereas 8- and 16-node SOMs produce nodes with overly 430 similar circulation patterns (ED_Figs. 1c and 2). Examination of the 4-, 8-, and 16-node 431 SOMs largely verifies the conclusions drawn from the 4-node SOM: that the occurrence 432 of patterns with ridging over the eastern half of the domain has increased over time, while 433 the occurrence of complimentary patterns has decreased. We note that these pattern 434 trends are not identified in the 2-node SOM, confirming that two nodes are too few to 435 capture specific circulation patterns that are critical for extreme temperature occurrence.

436 Similar node-count analyses for other regions/seasons likewise verify the conclusions 437 drawn from the 4-node analyses that are presented in the main text (not shown).

\section{Calculation of Robust Trends In Circulation Patterns}

$440 \quad$ For each season in each year, we calculate (1) the total number of days on which 441 each SOM pattern occurs ("occurrence"; [d•yr $\left.{ }^{-1}\right]$ ), (2) the mean length of consecutive 442 occurrence ("persistence"; [d•event $\left.{ }^{-1}\right]$ ), and (3) the longest consecutive 443 occurrence ("maximum duration"; $\left[\right.$ deevent $\left.\left.{ }^{-1}\right]\right)$. A trend in one of these characteristics is 444 considered robust when the trend in that pattern is (1) statistically significant in all three 445 reanalyses, and (2) of the same sign in all three reanalyses. Trends with 446 regression coefficients that surpass the 5\% significance (95\% confidence) threshold are 447 considered statistically significant. Trends are calculated across sat-era and ice-era annual 448 time-series using the approach of Zwiers and von Storch (37), which allows us to account 449 for temporal dependence. Here the trends are calculated using linear least squares 450 regression, but to account for temporal dependence, the confidence bounds of annual 451 time-series trends with lag-1 autocorrelation greater than the 5\% significance level are 452 recalculated by adjusting the number of degrees of freedom used to compute the 453 regression coefficient significance ${ }^{37}$. As a result, unlike a simple linear regression, this 454 approach does not rely on the independent and identically distributed ("i.i.d.") 455 assumption for the residuals, but instead accounts for temporal dependence using a red 456 noise assumption.

457 The approach of ref. 37 also assumes that the distribution of the residuals is 458 Gaussian. Using the Anderson-Darling ${ }^{38}$ test for normality, we find that $91-100 \%$ of 459 residual distributions in each metric of each reanalysis do not reject the null hypothesis of 
460 Gaussianity when multiple hypothesis testing controlling the Familywise Error Rate ${ }^{39}$ 461 (FWER) at the 5\% significance level is considered (ED_Table 1b). The Gaussianity 462 assumption is therefore largely appropriate. However, due to the identification of non463 normality in some distributions, particularly in the persistence and maximum duration 464 metrics, we apply Box-Cox power transformations ${ }^{40}$ to all distributions. Using the 465 Anderson-Darling test, we find that 96-100\% of the distributions of the residuals in the 466 transformed setting do not reject the null hypothesis of Gaussianity at the 5\% significance 467 level. Further, when multiple hypothesis testing is considered by controlling the FWER at 468 the $5 \%$ level, $100 \%$ of the individual tests are non-significant (ED_Table 1b). In addition,

469 the number of pattern trends identified as significant in the transformed case is largely 470 consistent with the non-transformed regression analysis. However, additional significant 471 trends in the persistence and maximum duration metrics are identified when Box-Cox 472 transformations are used (ED_Table 1a). The large overlap of results between the 473 transformed and non-transformed analyses suggests that although individual residual 474 distributions may vary, the Gaussian assumption applied throughout this study is for the 475 most part quite robust, though in some cases not valid. In sum, the non-transformed 476 analysis that fits a linear relationship to circulation pattern metrics allows for a relatively 477 simple classification of two short analysis periods, while simultaneously accounting for 478 temporal dependence in a large number $(>2000)$ of individual time-series $(7$ regions $\times 4$ 479 nodes $\times 4$ seasons $\times 3$ characteristics $\times 2$ time periods $\times 3$ reanalyses).

$480 \quad$ Because SOM nodes are calculated independently for each reanalysis, individual 481 SOM patterns must be matched between the three reanalyses in order to determine 482 whether an individual pattern shows robust results across all three reanalyses. To 483 determine which SOM patterns are the closest match between the three reanalyses, the 484 root mean square error (RMSE) is calculated between the SOM patterns of one reanalysis 485 and those of the other reanalyses. Patterns with the smallest RMSEs are considered 486 matches. Although we undertake a multi-reanalysis robustness evaluation, further work is 487 needed to confirm that other available reanalyses (such as CFSR and MERRA) show the 488 same trends. 
It is possible that some of the trends identified as significant in any individual reanalysis could occur by chance. In addition to screening for those patterns that are 493 significant in all three reanalyses (our "robustness" criterion), we also employ formal 494 multi-hypothesis testing utilizing several methodologies. The first is the familywise error 495 rate (FWER). This type of error metric controls the probability of falsely rejecting any 496 null hypothesis, and is considered one of the strictest forms of error control ${ }^{39}$. Since a 497 certain number of false rejections can happen by chance alone, one can account for this 498 formally by using the k-FWER or k-familywise error rate ${ }^{41}$ (k-FWER). The k-FWER 499 controls the probability of falsely rejecting $\mathrm{k}$ or more null hypotheses, and aims to 500 formalize the concept that some of the hypotheses will be rejected by chance. One option 501 for the value of $\mathrm{k}$ is to use the expected number of hypotheses that will be rejected at a 502 given significance level. For instance, in our study, out of 112 total "local" hypotheses, 5 503 or 6 hypothesis will be significant at the $5 \%$ significance level by chance $(112 \times 0.05=$ 504 5.6). In this case, one can evaluate the probability that 7 or more hypothesis are falsely 505 rejected, since on average about 6 could be rejected as significant by chance. The third 506 metric is the false discovery rate (FDR), which controls the expectation of the ratio given 507 by the number of false rejections divided by the total number of rejections ${ }^{39}$.

508 All of the above measures of error control aim to guard against hypotheses being 509 falsely declared as significant in the context of multiple tests. To be thorough, we have 510 implemented all three types of error control at both global significance levels of $5 \%$ and 511 10\%. The results of these analyses are summarized in Extended Data Table 2. We note 512 that all three metrics heavily favor the null as they are designed to protect against the 513 possibility of false positives. Despite this, the presence of local tests that reject the null 514 represents a strong confirmation of the significance of those local tests. The fact that a 515 number of local hypotheses still prevail as significant, even after imposing much stricter 516 multiple testing error controls, arises partly from the fact that some of the local p-values

517 indicate trends that are so highly significant that they can withstand the stricter multiple 518 testing error control metrics. We believe that this rigorous multiple-testing error control 519 yields increased credibility to the scientific conclusions of robust trends in pattern 520 occurrence.

521 


\section{Temperature Extremes}

523 Daily-scale hot and cold extreme occurrences are calculated using temperature

524 anomalies at each grid cell. Temperature anomalies are computed by removing the

525 seasonal cycle from daily reanalysis 2 -meter maximum/minimum temperatures. Similar

526 to previous studies ${ }^{1,2}$, temperature extremes are calculated based on the statistical

527 distribution of daily temperature anomalies ${ }^{20}$. Hot/cold extreme thresholds are defined as

528 the $95^{\text {th }} / 5^{\text {th }}$ percentile value of the 1979-2013 daily 2-meter maximum/minimum

529 temperature anomaly distribution (e.g., for JJA, the population of daily-maximum

530 temperature anomalies from the months of June, July and August in the years 1979-

531 2013). Hot/cold extreme occurrences are defined as days on which the daily temperature

532 anomalies are greater/less than (or equal to) the hot/cold extreme thresholds. Reanalysis

533 temperature extremes are qualitatively similar to those found in station-based

534 observations $^{2}$. Given this similarity, we use the reanalysis temperatures in order to

535 maintain internal physical consistency between daily 2-meter temperatures and daily

536 atmospheric circulation (as represented by the $500 \mathrm{hPa}$ SOM circulation patterns). Trends

537 in temperature extreme occurrence are computed across sat-era and ice-era annual time-

538 series following the methodology of ref. 37.

539

\section{Quantitative Partitioning}

541 To determine the dynamic and thermodynamic contributions to trends in 542 temperature extreme occurrence, we adapt the climate change partitioning methodology

543 of Cassano et al (10). Our adapted methodology partitions the contributions of dynamic

544 and thermodynamic changes to (1) the overall trend in temperature extreme occurrence

545 and (2) the trends associated with individual SOM circulation patterns. Previous

546 applications of the Cassano et al methodology indicate that partitioning is largely

547 insensitive to the number of SOM nodes used in the analysis ${ }^{42}$. All trends in temperature

548 extremes in the below methodology are area-weighted averages. Following Cassano et al:

$$
E=\sum_{i=1}^{K} E_{i} f_{i}
$$

549 where $E$ is the frequency of extreme temperature occurrence, $f_{i}$ is the frequency of 550 occurrence of SOM pattern $i, E_{i}$ is the frequency of extreme temperature occurrence 
551 when SOM pattern $i$ occurs, and $K$ is the total number of SOM nodes. We decompose $E$ 552 and $f$ into time mean and deviation from time mean components:

$$
E=\sum_{i=1}^{K}\left(\bar{E}_{l}+E_{i}{ }^{\prime}\right)\left(\overline{f_{l}}+f_{i}{ }^{\prime}\right)
$$

553 Now we differentiate the above equation with respect to time, noting that the mean values 554 are constants:

$$
\frac{d E}{d t}=\sum_{i=1}^{K}\left(\bar{f}_{l} \frac{d E_{i}{ }^{\prime}}{d t}+\bar{E}_{l} \frac{d f_{i}{ }^{\prime}}{d t}+\frac{d}{d t}\left(E_{i}{ }^{\prime} f_{i}{ }^{\prime}\right)\right)
$$

555 The derivative on the left-hand side provides the area-weighted average trend in 556 the seasonal occurrence of temperature extremes for all days. The summation on the 557 right-hand side, from left to right, provides the thermodynamic, dynamic, and interaction 558 contributions for days associated with each SOM pattern, $i$.

559 The thermodynamic contribution of each circulation pattern's extreme 560 temperature trend assumes that each SOM pattern is stationary in time, and that trends in 561 extremes that result during this pattern are the result of influences unrelated to 562 circulation, such as changes in longwave radiation from increasing greenhouse gas 563 concentrations, or changes in surface fluxes of moisture and/or radiation resulting from 564 changes in land cover. The thermodynamic contribution associated with each circulation 565 pattern is determined by taking the product of the trend in the intensity of temperature 566 extremes and the mean occurrence of the circulation pattern. Trends in the intensity of 567 temperature extremes are computed by calculating the trend in area-weighted extreme 568 occurrence per pattern occurrence (e.g., Fig. 2n-q).

569 The dynamic contribution of each circulation pattern's extreme temperature trend 570 assumes that, on average, a circulation pattern is associated with a portion of the total 571 extreme event trend, and that changes in the occurrence frequency of that circulation 572 pattern will modify the occurrence frequency of extreme events. The dynamic 573 contribution associated with each circulation pattern is determined by taking the product 574 of the trend in circulation pattern occurrences and the mean number of extreme events per 575 pattern occurrence.

576 The third component represents the interaction between dynamic and 577 thermodynamic changes, and captures contributions that result from changes in the 
578 dynamic component acting on changes in the thermodynamic component, such as the

579 positive/negative feedbacks of surface-atmosphere interactions. The interactive term is

580 determined by computing the trend in the product of circulation pattern occurrence

581 deviations and intensity of temperature extreme deviations.

582

583 Code Availability

584 SOM code is available at: http://www.cis.hut.fi/projects/somtoolbox/. All other

585 analysis code is available upon request from the corresponding author via email:

586 danethan@stanford.edu.

587

588 Method References

589

590 31. Screen, J. A. \& Simmonds, I. Amplified mid-latitude planetary waves favour

591 particular regional weather extremes. Nature Clim. Change 4, 704-709 (2014).

592

593 32. Kalnay, E., et al. The NCEP/NCAR 40-year reanalysis project, Bull. Am. Meteorol.

594 Soc., 83, 437-471 (1996).

595

596 33. M. Kanamitsu, W. et al. NCEP-DOE AMIP-II Reanalysis (R-2). Bull. Am. Meteorol.

597 Soc. 83, 1631-1643 (2002).

598

599 34. Dee, D. P. et al. The ERA-Interim reanalysis: configuration and performance of the

600 data assimilation system. Q. J. R. Meteorol. Soc. 137, 553-597 (2011).

601

602 35. Driouech, F., Déqué M. \& Sánchez-Gómez, E. Weather-regimes-Moroccan

603 precipitation link in a regional climate change simulation. Glob. Plan. Ch. 72, 1-10

604 (2010).

605

606 36. Cattiaux, J., Douville, H. \& Peings, P. European temperatures in CMIP5: origins of 607 present-day biases and future uncertainties. Clim. Dyn. 41 2889-2907 (2013).

608 
610 mean. J. Clim. 8, 336-351 (1995).

611

612 38. Anderon, T.W. \& Darling, D.A. Asymptotic theory of certain "goodness-of-fit"

613 criteria based on stochastic processes. Ann. Math. Statist. 23, 193-212 (1952).

614

615 39. Benjamini, Y. \& Hochberg, Y. Controlling the false discovery rate: A practical and

616 powerful approach to multiple testing. J. Roy. Statist. Soc. Ser. B 57, 289-300 (1995).

617

618 40. Box, G.E.P. \& Cox, D.R. An analysis of transformations. J. Roy. Statist. Soc. Ser. B

619 26, 211-243 (1964).

620

621 41. Lehmann, E. \& Romano, J. P. Generalizations of the familywise error rate. The

622 Annals of Statistics 33, 1138-1154 (2005).

623

624 42. Skific, N., Francis, J. A. \& Cassano, J. J. Attribution of projected changes in

625 atmospheric moisture transport in the Arctic: A self-organizing map perspective. J. Clim.

626 22, 4135-4153 (2009).

627

628 Data

629 Reanalysis Datasets

630 NCAR/NCEP-Reanalysis 1 data downloaded from: www.esrl.noaa.gov/psd/

631 NCEP-DOE-Reanalysis 2 data downloaded from: www.esrl.noaa.gov/psd/

632 ECMWF ERA-Interim data downloaded from: www.ecmwf.int/

633

634 Extended Data Figure \& Table Legends:

635

636 Extended Data Figure 1 | 2-, 4-, and 8-node SOM analyses. SOM-derived mid637 atmospheric summer (JJA) circulation patterns ( $500 \mathrm{hPa}$ geopotential height anomalies)

638 over Europe using a, 2-, b, 4-, and c, 8-node analyses. White-boxed values show pattern

639 frequencies (\%) in the top left, and SOM node numbers in the top right. Time-series of 


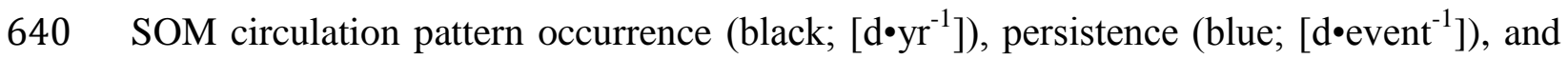
641 maximum duration (red; $\left[\mathrm{d}^{\bullet} \mathrm{event}^{-1}\right]$ ). The slope of the trend line $\left[\mathrm{yr}^{-1}\right]$ and $\mathrm{p}$-values (in 642 parentheses) are color coded, with the values from 1979-2013 (solid trend line) displayed 643 above those from 1990-2013 (dashed trend line). Statistically significant trends (5\% 644 significance level) are shown by bold fonts in the scatter plots. Geopotential height fields 645 are sourced from the NCEP-DOE-R2 reanalysis ${ }^{33}$.

647 Extended Data Figure 2 | 16-node SOM analysis. SOM-derived mid-atmospheric 648 summer (JJA) circulation patterns (500 hPa geopotential height anomalies) over Europe 649 derived from a 16-node analysis. White-boxed values show pattern frequencies (\%) in the 650 top left, and SOM node numbers in the top right. Time-series of SOM circulation pattern 651 occurrence (black; $\left[\mathrm{d} \bullet \mathrm{yr}^{-1}\right]$ ), persistence (blue; $\left[\mathrm{d} \bullet e^{-1} \mathrm{ent}^{-1}\right]$ ), and maximum duration (red; $\left.652\left[\mathrm{~d} \cdot \mathrm{event}^{-1}\right]\right)$. The slope of the trend line $\left[\mathrm{yr}^{-1}\right]$ and $\mathrm{p}$-values (in parentheses) are color 653 coded, with the values from 1979-2013 (solid trend line) displayed above those from 654 1990-2013 (dashed trend line). Statistically significant trends (5\% significance level) are 655 shown by bold fonts in the scatter plots. Geopotential height fields are sourced from the 656 NCEP-DOE-R2 reanalysis ${ }^{33}$.

658 Extended Data Figure 3 | Geopotential height anomaly trends and thermal dilation 659 adjustment. a-d, Northern Hemisphere polar projections of 1979-2013 seasonal trends in $660500 \mathrm{hPa}$ geopotential height anomalies (same as Fig.1, reproduced here for convenience).

661 e, Area-weighted trends in seasonal geopotential height anomalies over the Northern 662 Hemisphere and regional SOM domains. f-j, Trends in raw and detrended geopotential 663 height SOM pattern occurrence (OCC), persistence (PER), and maximum duration 664 (DUR) in units of ${\mathrm{d} \cdot \mathrm{yr}^{-2}}^{2}$ for domains and seasons highlighted in the main text. The 665 magnitudes of the (removed) seasonal Northern Hemisphere trends can be found in $\mathbf{e}$. 666 Grid cells highlighted in gray contain trends significant at the 5\% level. SOM circulation 667 patterns are abbreviated by letter: A-Anticyclonic, C-Cyclonic, and combinations of the 668 two represent dipole patterns and east-west configurations. Geopotential height fields are 669 sourced from the NCEP-DOE-R2 reanalysis ${ }^{33}$.

670 
671 Extended Data Figure 4 | 1979-2013 (sat-era) robust atmospheric circulation pattern

672 trends. Time-series of circulation pattern occurrence (black; [d• $\left.\mathrm{yr}^{-1}\right]$ ), persistence (blue;

$673\left[\mathrm{~d} \bullet \mathrm{event}^{-1}\right]$ ), and maximum duration (red; [d•event $\left.{ }^{-1}\right]$ ) from the NCEP-DOE-R2

674 reanalysis $^{33}$ : a, summer over Europe; b, summer over western Asia; c, summer over

675 eastern North America; d, autumn over eastern Asia; e, autumn over western Asia; f,

676 autumn over central North America; $\mathbf{g}$, autumn over eastern North America; and $\mathbf{h}$, spring

677 over Europe. Statistically significant trends $\left(\left[\mathrm{yr}^{-1}\right] ; 5 \%\right.$ significance level) are identified

678 by bold fonts in the scatter plots.

679

680 Extended Data Figure 5 | 1990-2013 (ice-era) robust atmospheric circulation pattern

681 trends. Time-series of circulation pattern occurrence (black; $\left[\mathrm{d} \bullet \mathrm{yr}^{-1}\right]$ ), persistence (blue; $682\left[\mathrm{~d} \bullet e^{-1} \mathrm{t}^{-1}\right]$ ), and maximum duration (red; [d•event $\left.{ }^{-1}\right]$ ) from the NCEP-DOE-R2 683 reanalysis ${ }^{33}$ : a, winter over western Asia; $\mathbf{b}$, winter over central Asia; $\mathbf{c}$, summer over 684 western Asia; d, summer over eastern North America; e, autumn over western Asia; and 685 f, autumn over eastern North America. Statistically significant trends ([yr $\left.{ }^{-1}\right] ; 5 \%$ 686 significance level) are identified by bold fonts in the scatter plots.

688 Extended Data Figure 6 | 1979-2013 (sat-era) Northern Hemisphere extreme 689 temperature occurrence trends. Sat-era extreme temperature trends $\left[\mathrm{d} \bullet \mathrm{yr}^{-1} \cdot \mathrm{yr}^{-1}\right]$ for 690 winter cold (a) and hot (b) occurrences; spring cold (c) and hot (d) occurrences; summer 691 cold (e) and hot (f) occurrences; and autumn cold (g) and hot (h) occurrences. Trends are 692 calculated from the NCEP-DOE-R2 reanalysis 2-meter daily maximum/minimum 693 temperatures ${ }^{33}$. Grid cells with statistically significant trends (5\% significance level) are 694 stippled.

695

696 Extended Data Figure 7 | 1990-2013 (ice-era) Northern Hemisphere extreme 697 temperature occurrence trends. Ice-era extreme temperature trends $\left[\mathrm{d} \cdot \mathrm{yr}^{-1} \cdot \mathrm{yr}^{-1}\right]$ for 698 winter cold (a) and hot (b) occurrences; spring cold (c) and hot (d) occurrences; summer 699 cold (e) and hot (f) occurrences; and autumn cold (g) and hot (h) occurrences. Trends are 700 calculated from the NCEP-DOE-R2 reanalysis 2-meter daily maximum/minimum 
701 temperatures ${ }^{33}$. Grid cells with statistically significant trends (5\% significance level) are 702 stippled.

704 Extended Data Figure 8 | Sensitivity of pattern similarity to number of SOM nodes.

705 To determine an adequate number of SOM nodes, we follow a modified version of the methodology introduced by Lee and Feldstein (12), wherein the mean pattern correlation of all daily geopotential height anomaly fields and their matching SOM node patterns are computed for a suite of different SOM node counts $(3,4,5,6,7$, and 8), for all regions and all seasons (black dots). We also compute the maximum/minimum pattern correlation

710 of daily geopotential height anomaly fields with their matching SOM node pattern (red

711 dots) and the maximum/minimum SOM-pattern-to-SOM-pattern correlation (blue

712 triangles). The goal is to select an adequate number of nodes such that: (1) the mean

713 pattern correlation of all daily geopotential height anomaly fields is relatively large, (2)

714 the minimum pattern correlation of daily geopotential height anomaly fields is relatively

715 large, and (3) the maximum SOM-pattern-to-SOM-pattern correlation is relatively small.

716 Similar to Lee and Feldstein, we find that four SOM nodes are generally sufficient to

717 capture the different modes of atmospheric variability, but small enough that SOM

718 patterns depict distinct circulations. Geopotential height anomaly fields are sourced from 719 the NCEP-DOE-R2 reanalysis ${ }^{33}$.

721 Extended Data Table 1 | Significant reanalysis circulation pattern trends and 722 summary of multiple hypothesis testing. a, Here we indicate the number of linear 723 regression and Box-Cox transformed statistically significant occurrence (occ), persistence

724 (per), and maximum duration (max dur) trends that surpass the 95\% confidence threshold 725 (5\% significance level) for each reanalysis in each analysis period (1979-2013 = "sat-era" 726 and 1990-2013 = "ice-era"). For a given metric (occ, per and max dur) in a given period 727 (sat-era and ice-era), there are four patterns per region, over four seasons, for 7 domains. 728 There are therefore 112 total trends in each metric in each period. b, Results of 729 Anderson-Darling ${ }^{38}$ (AD) tests of normality indicating the number of original and Box730 Cox transformed ${ }^{40}$ distributions of residuals that do not reject the null hypothesis of 731 Gaussianity at the 5\% level. For both the original and Box-Cox AD tests, we also apply 
732 multiple hypothesis testing to control the Familywise Error Rate ${ }^{39}$ (FWER) at the 5\%

733 significance level. c, To protect against the possibility of false positives in the linear

734 regression trend analyses, for each reanalysis, we use three different multiple hypothesis

735 error control methodologies to assess the number of locally significant hypotheses that

736 surpass the global $5 \%$ or $10 \%$ significance level under strict error control requirements.

737 FWER $^{39}$, k-familywise error rate ${ }^{41}$ (k-FWER), and false discovery rate ${ }^{39}$ (FDR) analyses

738 are applied (Methods). We note that all three metrics heavily favor the null. The presence

739 of local tests that reject the null therefore represents a strong confirmation of the

740 significance of those local tests, as those local tests are so highly significant that they can

741 withstand the stricter multiple testing error control criteria.

742

743 Extended Data Table 2 | Quantitative partitioning of temperature extreme trends

744 for select SOM analyses. Trends are partitioned for a Europe, b western Asia, and c

745 eastern North America sat-era summer hot extremes; d central Asia ice-era winter cold

746 extremes; e eastern North America sat-era autumn hot extremes; and f eastern Asia sat-

747 era autumn cold extremes. From the left column moving right, (1) SOM node number, (2)

748 trend in pattern occurrence ["pat-occ" $\mathrm{d}^{\mathrm{yr}}{ }^{-1} \cdot \mathrm{yr}^{-1}$ ], (3) mean pattern occurrence for the

749 period ["pat-occ" $\mathrm{d} \cdot \mathrm{yr}^{-1}$ ], (4) trend in temperature extremes per pattern occurrence

750 (intensity of extreme occurrence) ["ext-occ" d•"pat-occ" $\left.\mathrm{d}^{-1} \cdot \mathrm{yr}^{-1} \cdot \mathrm{yr}^{-1}\right]$, (5) mean

751 temperature extreme per pattern occurrence ["ext-occ"d."pat-occ" $\left.{ }^{-1} \cdot \mathrm{yr}^{-1}\right]$, (6) trend in

752 the dynamic influences ["ext-occ" $\mathrm{d} \cdot \mathrm{yr}^{-1} \cdot \mathrm{yr}^{-1}$ ], (7) trend in the thermodynamic influences

753 ["ext-occ" $\left.d \cdot \mathrm{yr}^{-1} \cdot \mathrm{yr}^{-1}\right]$, (8) trend in the interaction of dynamic and thermodynamic

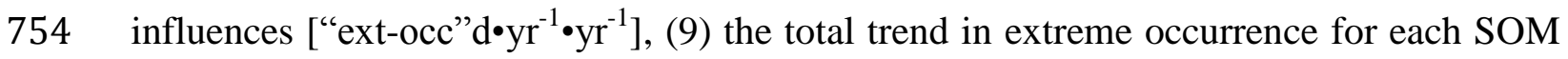

755 pattern ["ext-occ" $\left.d \cdot \mathrm{yr}^{-1} \cdot \mathrm{yr}^{-1}\right],(10)$ the percent of the total trend in extreme occurrences

756 that occur during each pattern (pattern trend percentages sum to $100 \%$, meaning

757 contributions from individual SOM patterns can be either positive or negative; that is,

758 trend percentages greater than $100 \%$ indicate that other circulation patterns provide

759 negative contributions), (11) the percent of column 10 that is due to dynamic influences,

760 (12) the percent of column 10 that is due to thermodynamic influences, and (13) the

761 percent of column 10 that is due to interactive influences (dynamic, thermodynamic, and

762 interactive influence percentages for individual SOM patterns sum to $100 \%$, meaning 
763 contributions from individual influences can be either positive or negative). The overall 764 trends for the domain are presented below the individual SOM rows. All data are sourced 765 from the NCEP-DOE-R2 reanalysis ${ }^{33}$. 


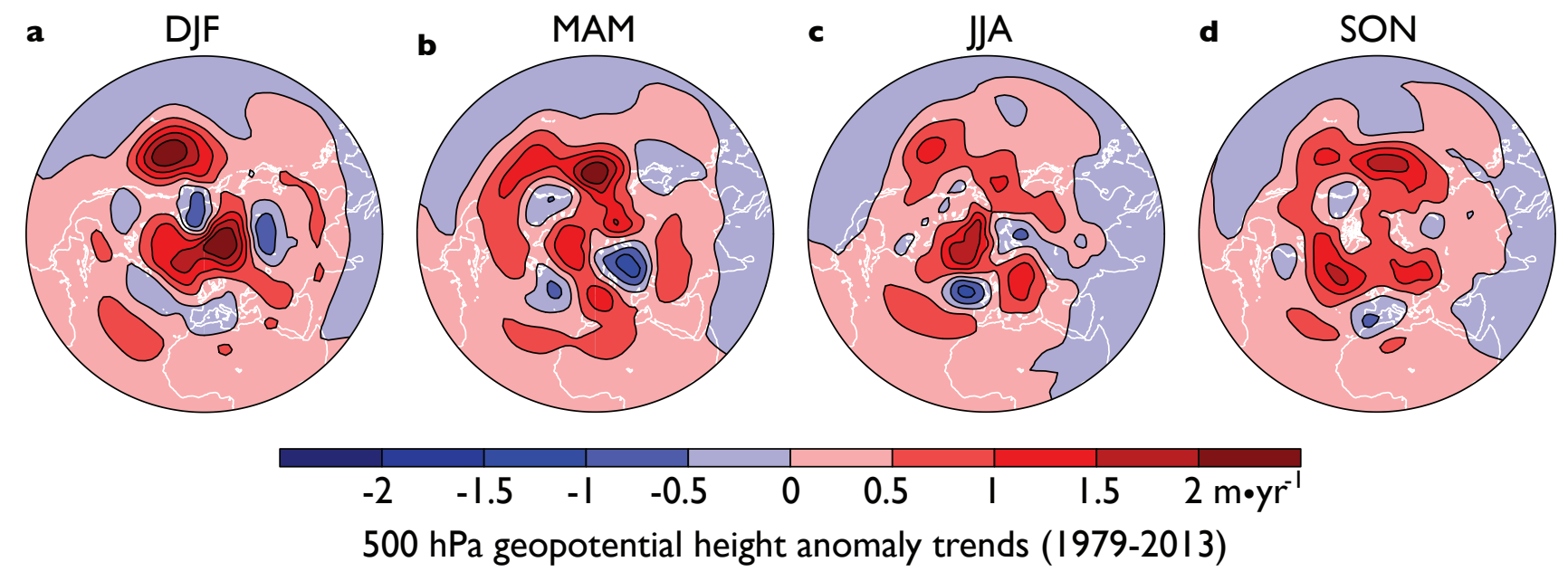




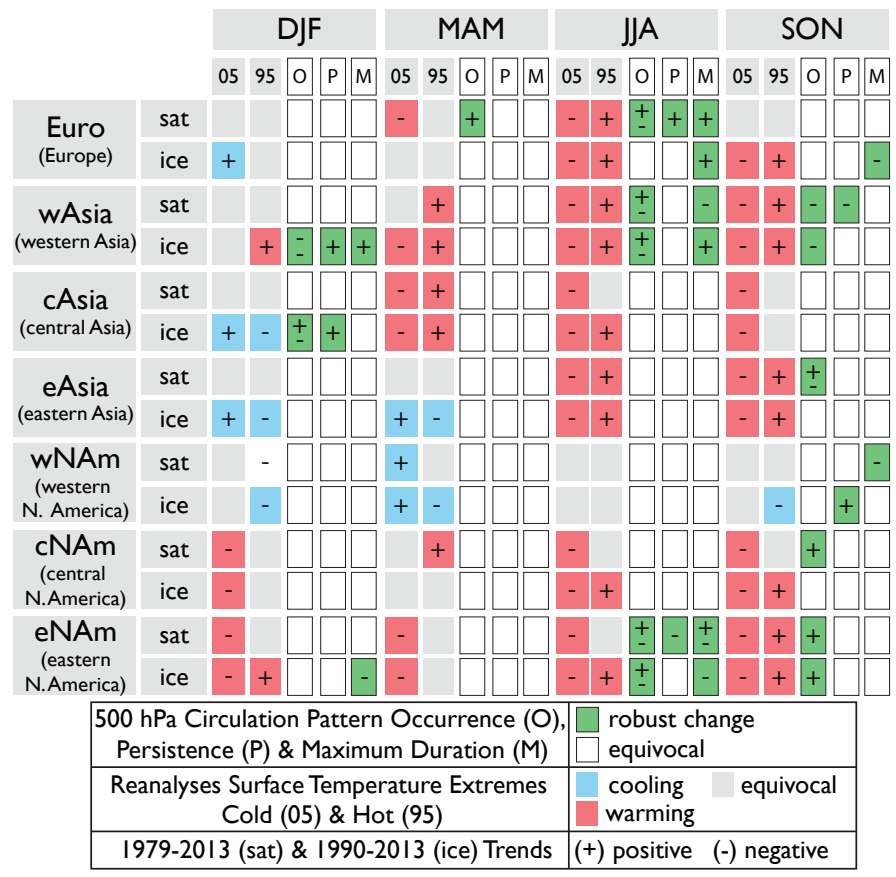



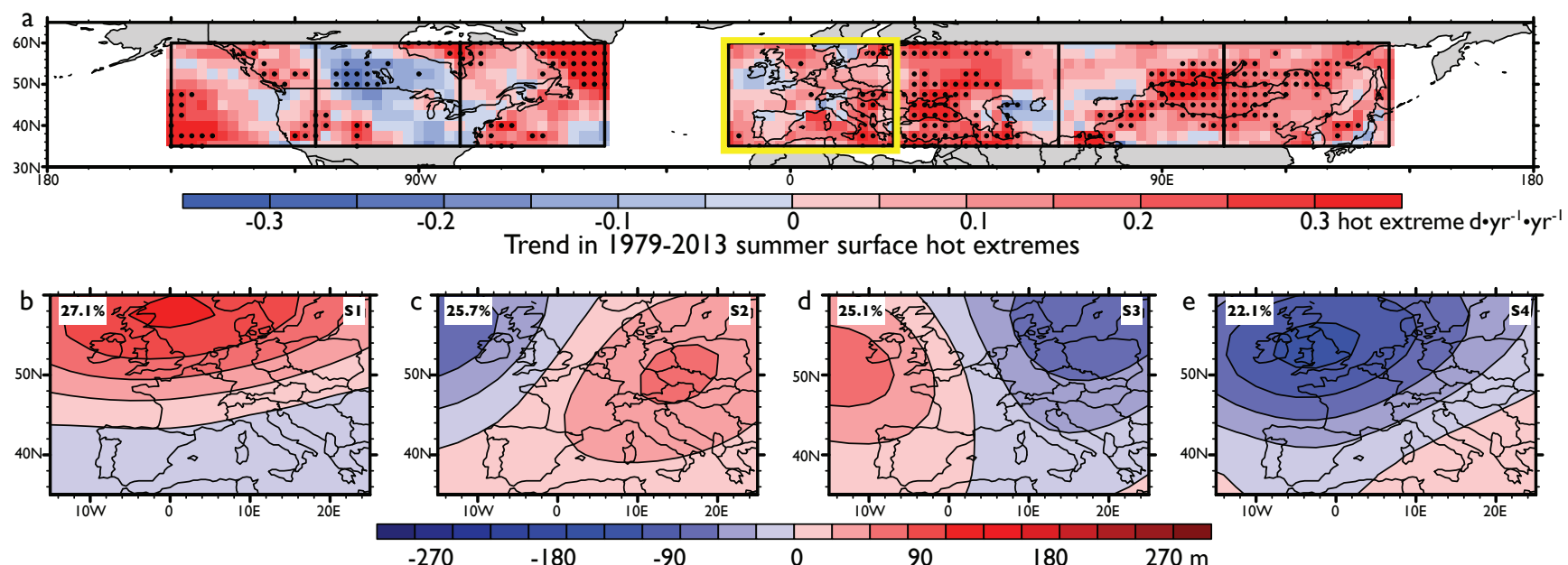

$\begin{array}{ccccc}-270 & -180 & -90 & 0 & 90 \\ & & 500 \mathrm{hPa} \text { circulation patterns }\end{array}$
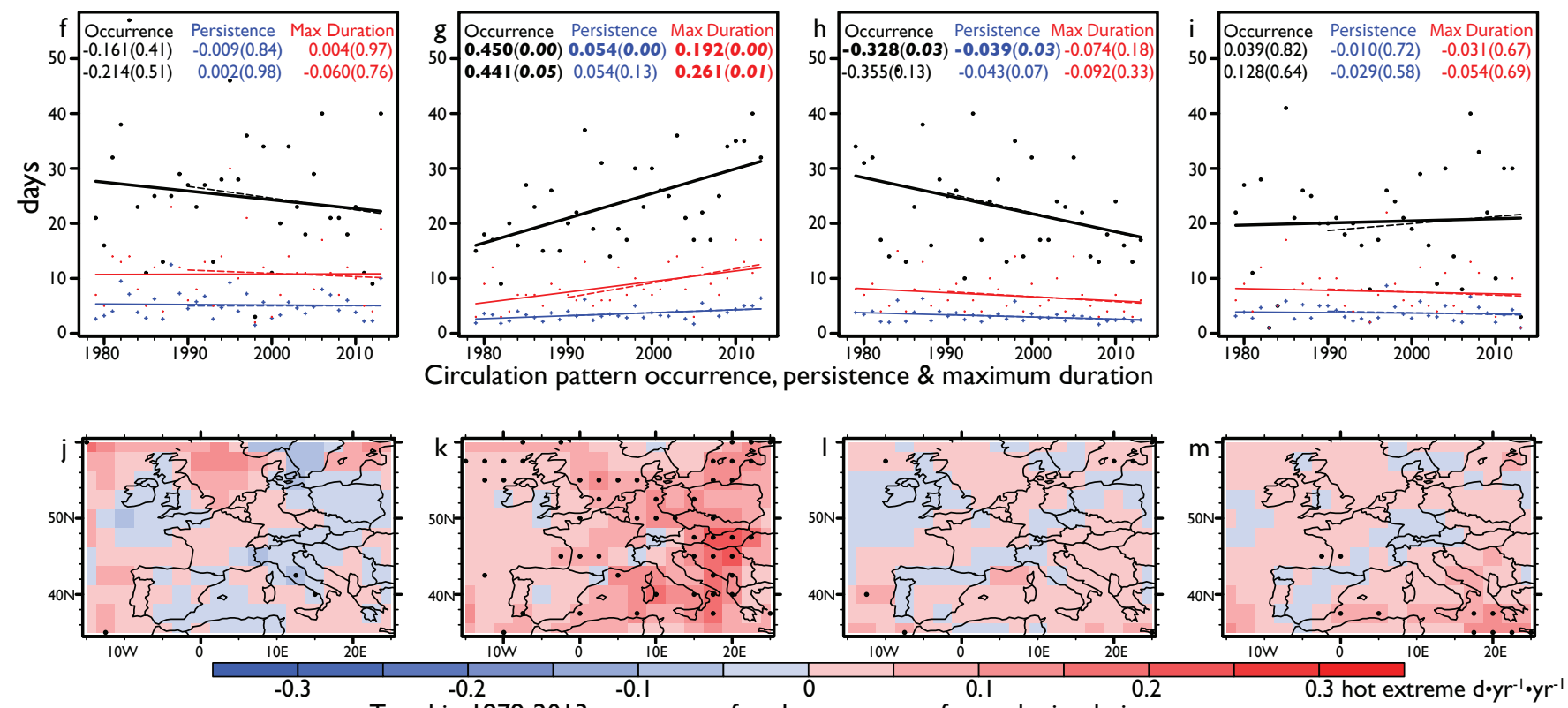

Trend in 1979-2013 summer surface hot extremes for each circulation pattern
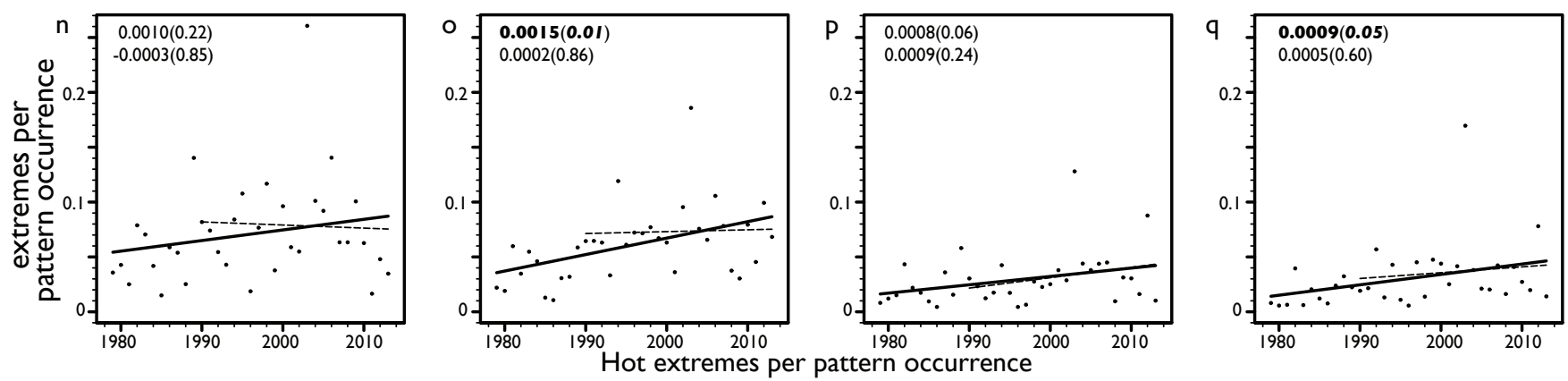

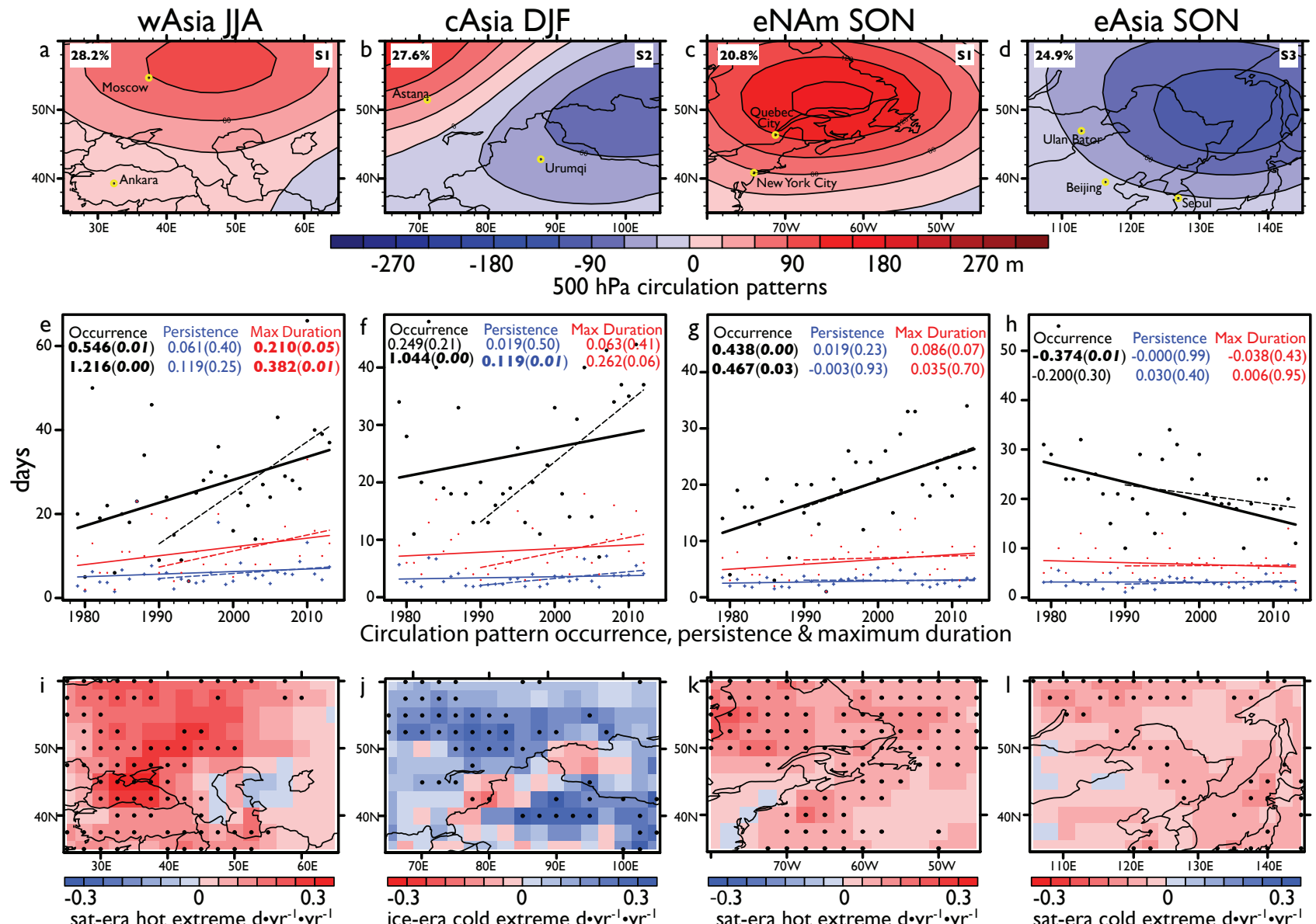

Trend in surface temperature extremes for each circulation pattern
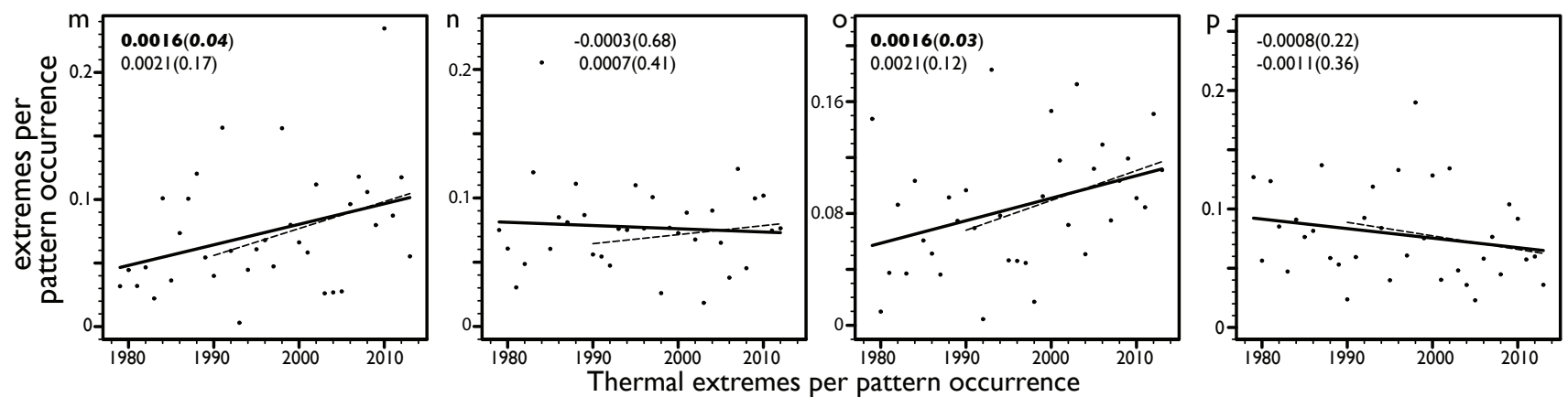

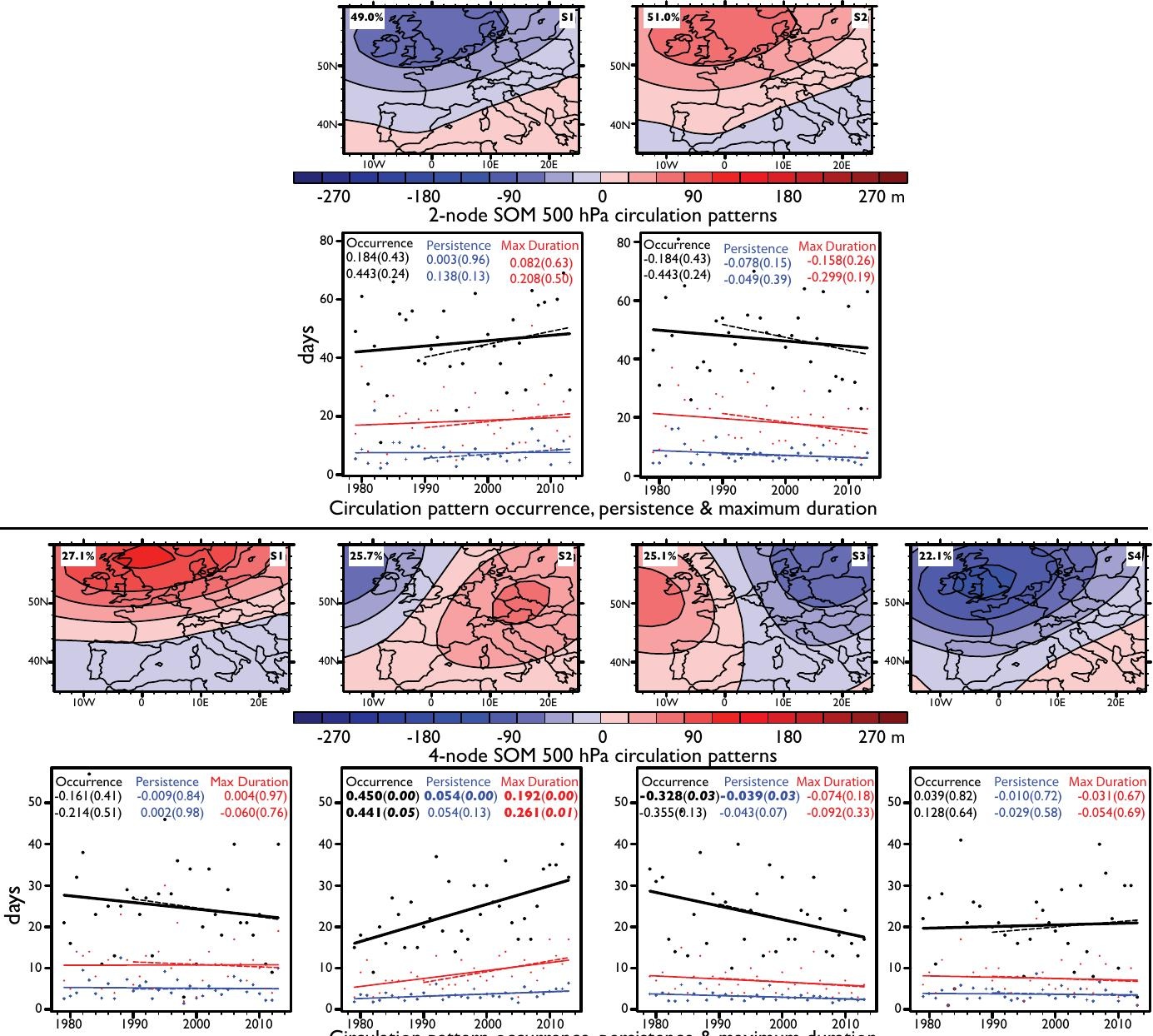

a circulation patterns
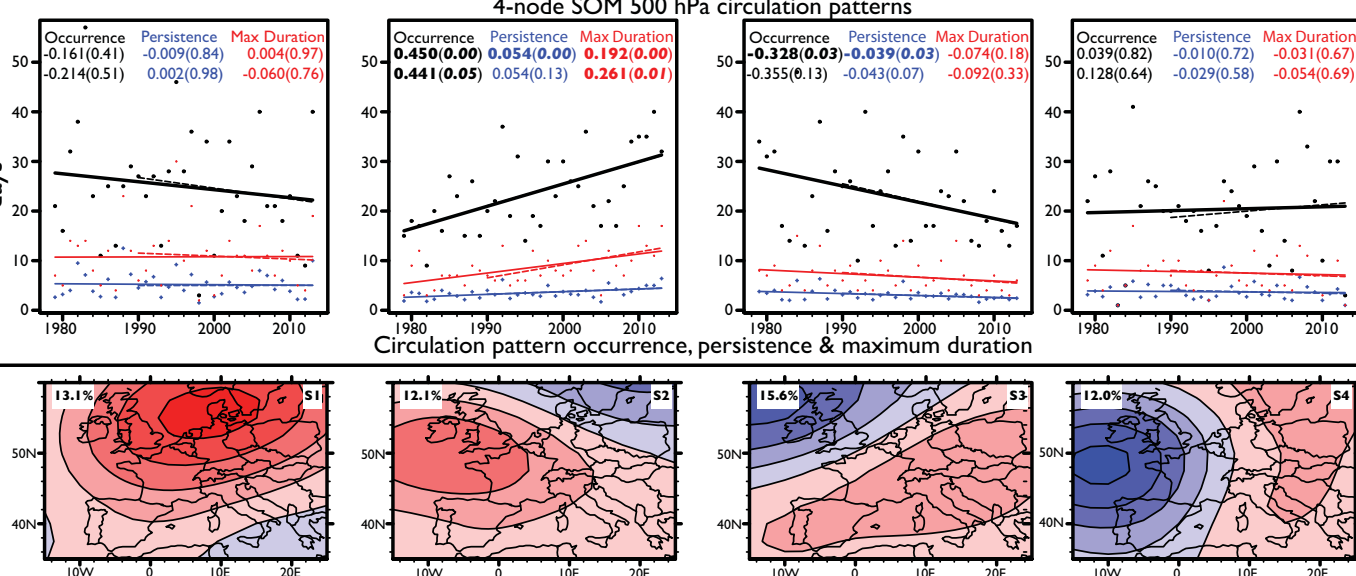

Circulation pattern occurrence, persistence \& maximum duration
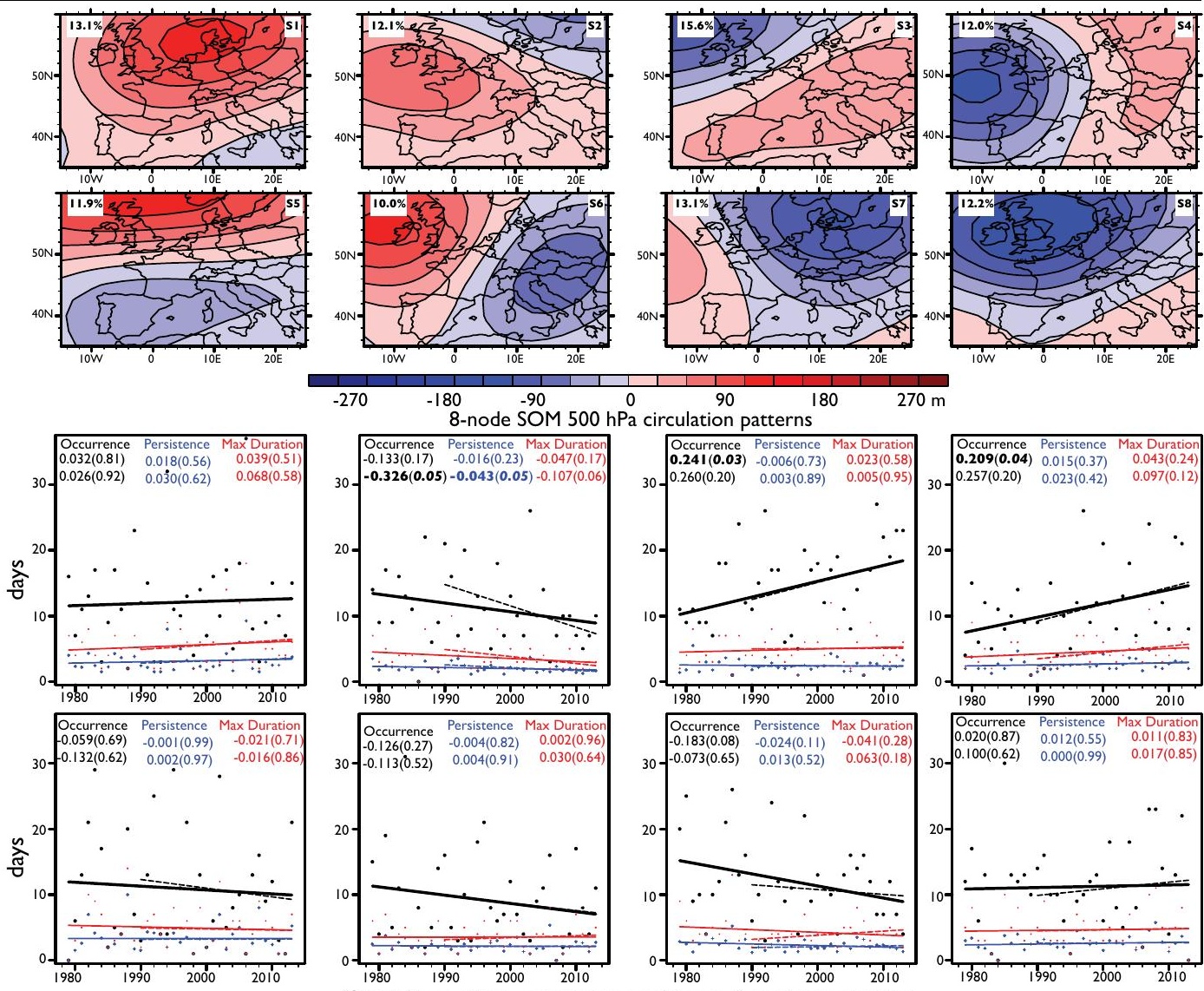

Circulation pattern occurrence, persistence \& maximum duration

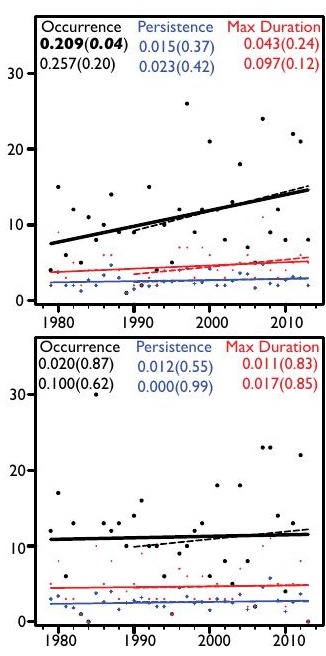



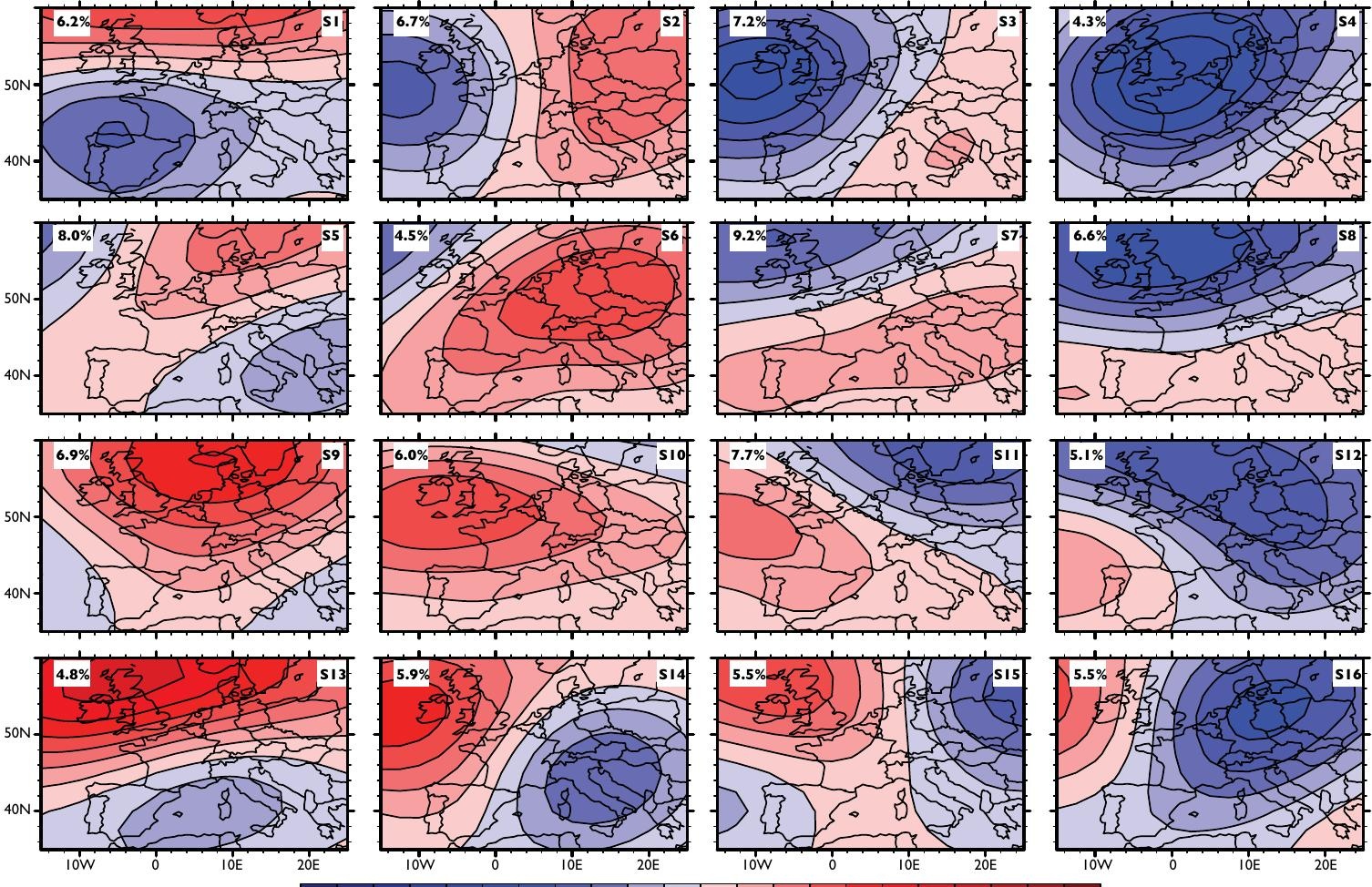

$-270$

$\begin{array}{lllll}-180 & -90 & 0 & 90 & 180\end{array}$

$270 \mathrm{~m}$
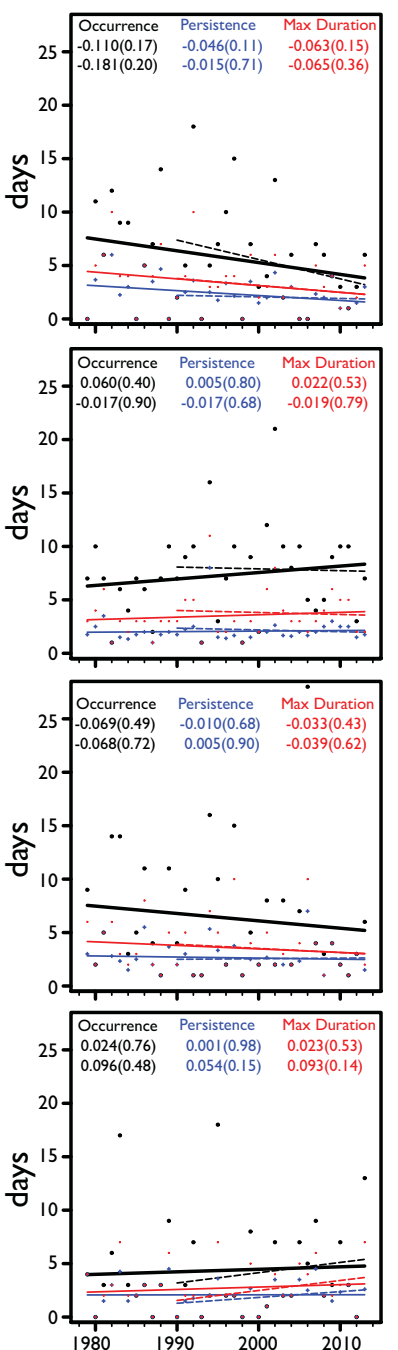
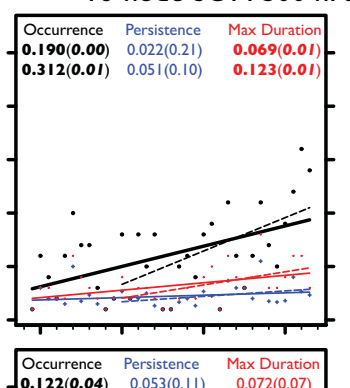

$\begin{array}{|ccc|}\mathbf{0 . 1 2 2 ( 0 . 0 4 )} & 0.053(0.11) & 0.072(0.07) \\ 0.013(0.91) & -0.004(0.96) & -0.005(0.95) \\ & & \end{array}$
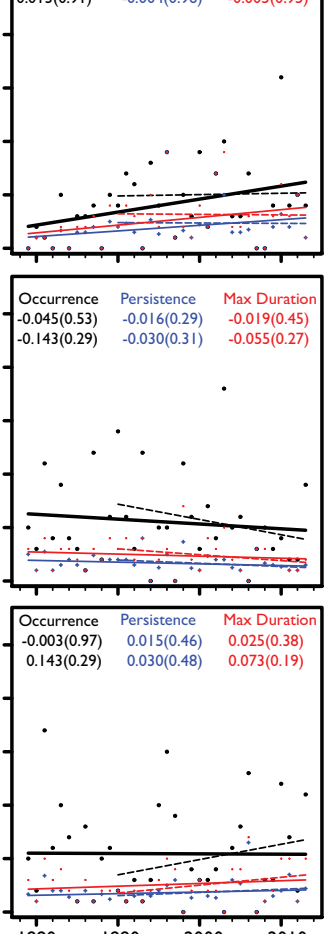

$1980 \quad 199020002010$

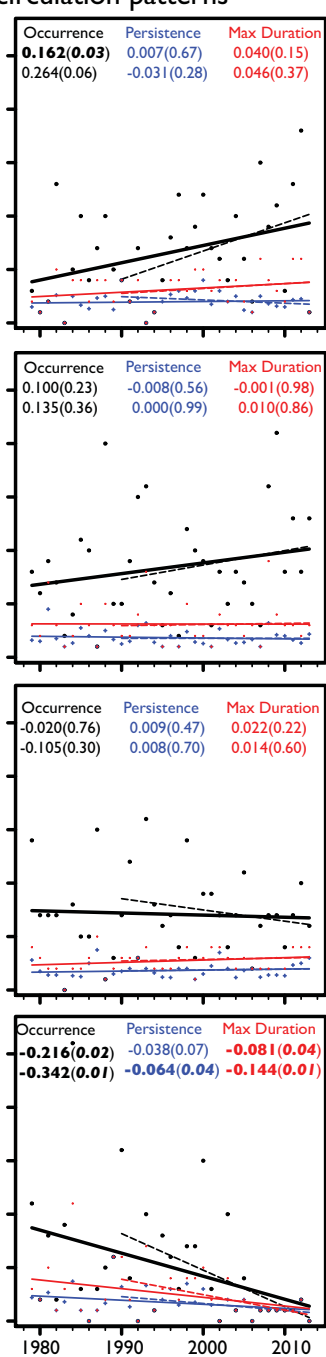

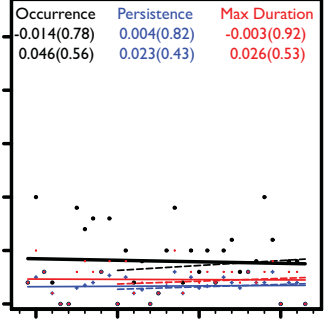
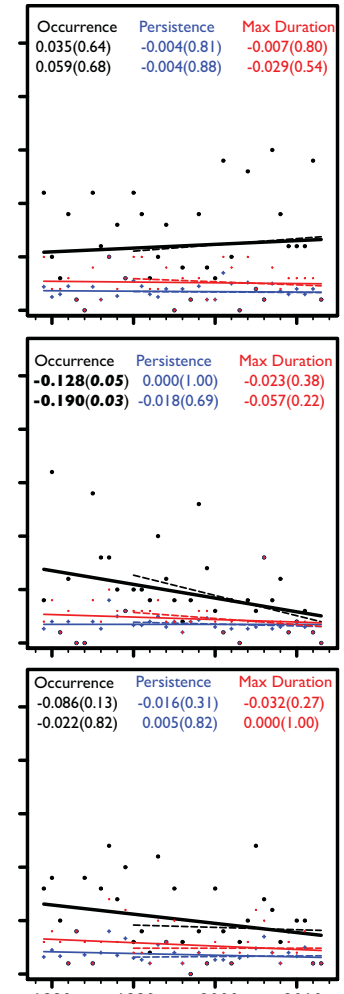

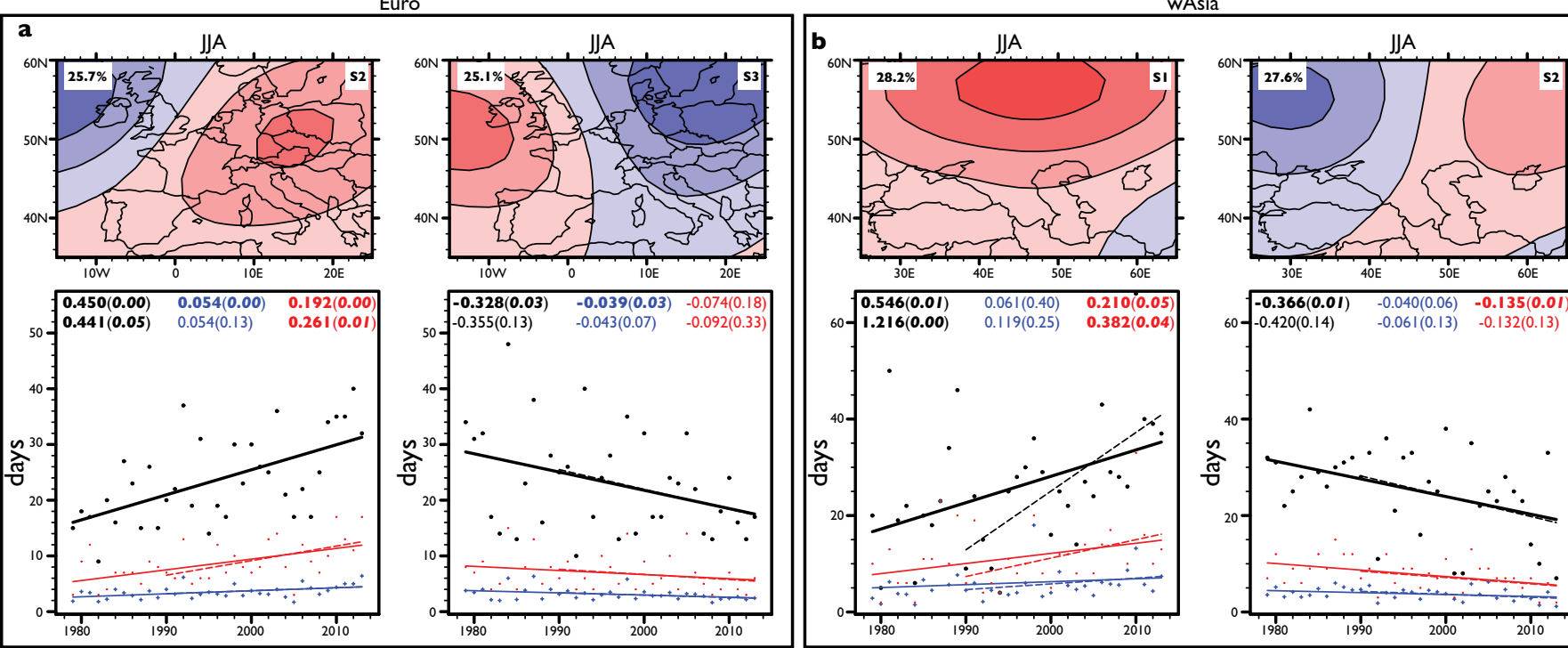

eNAm
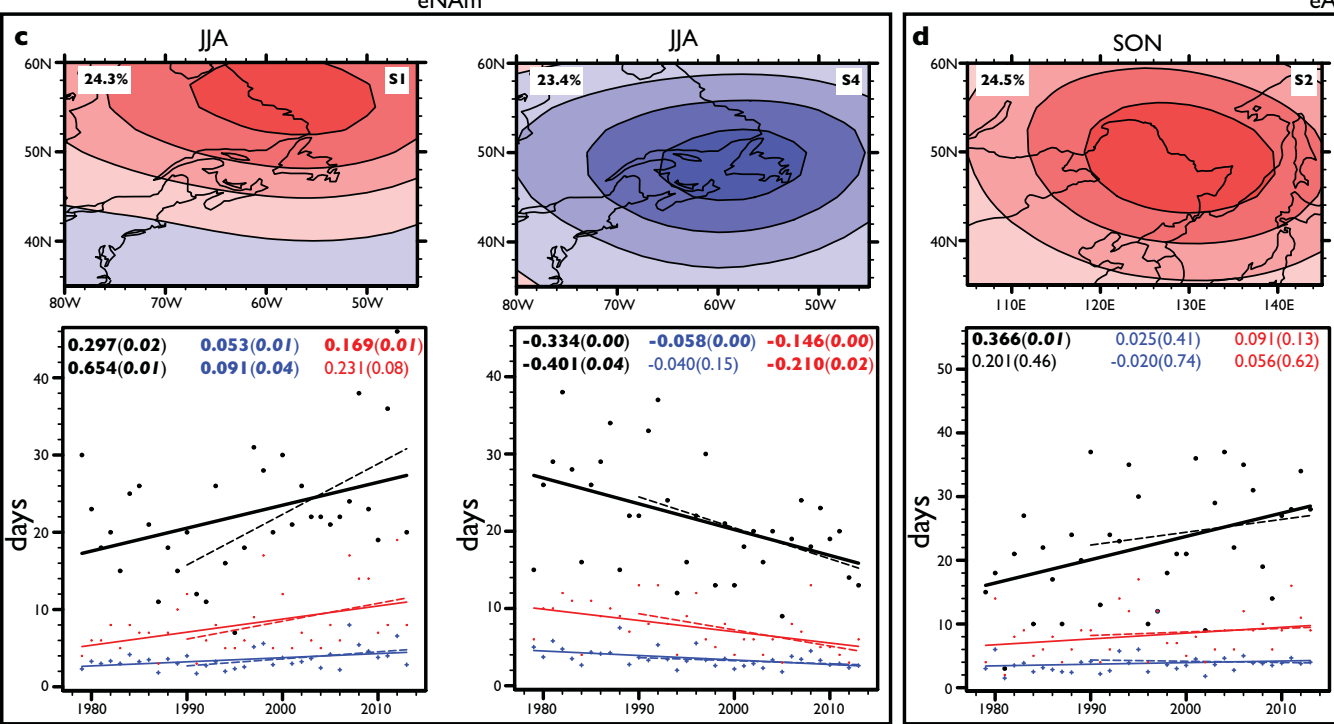

eAsia
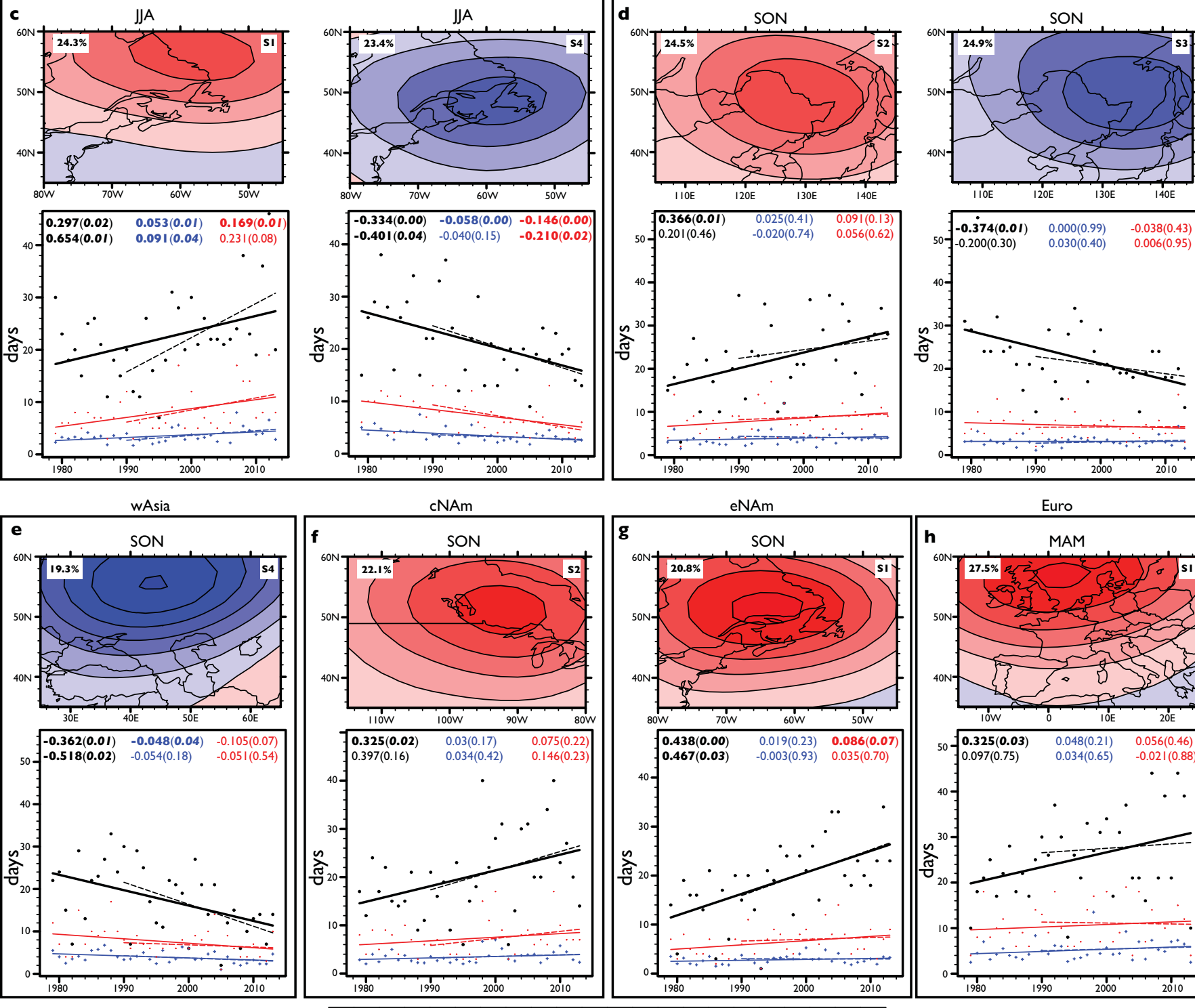

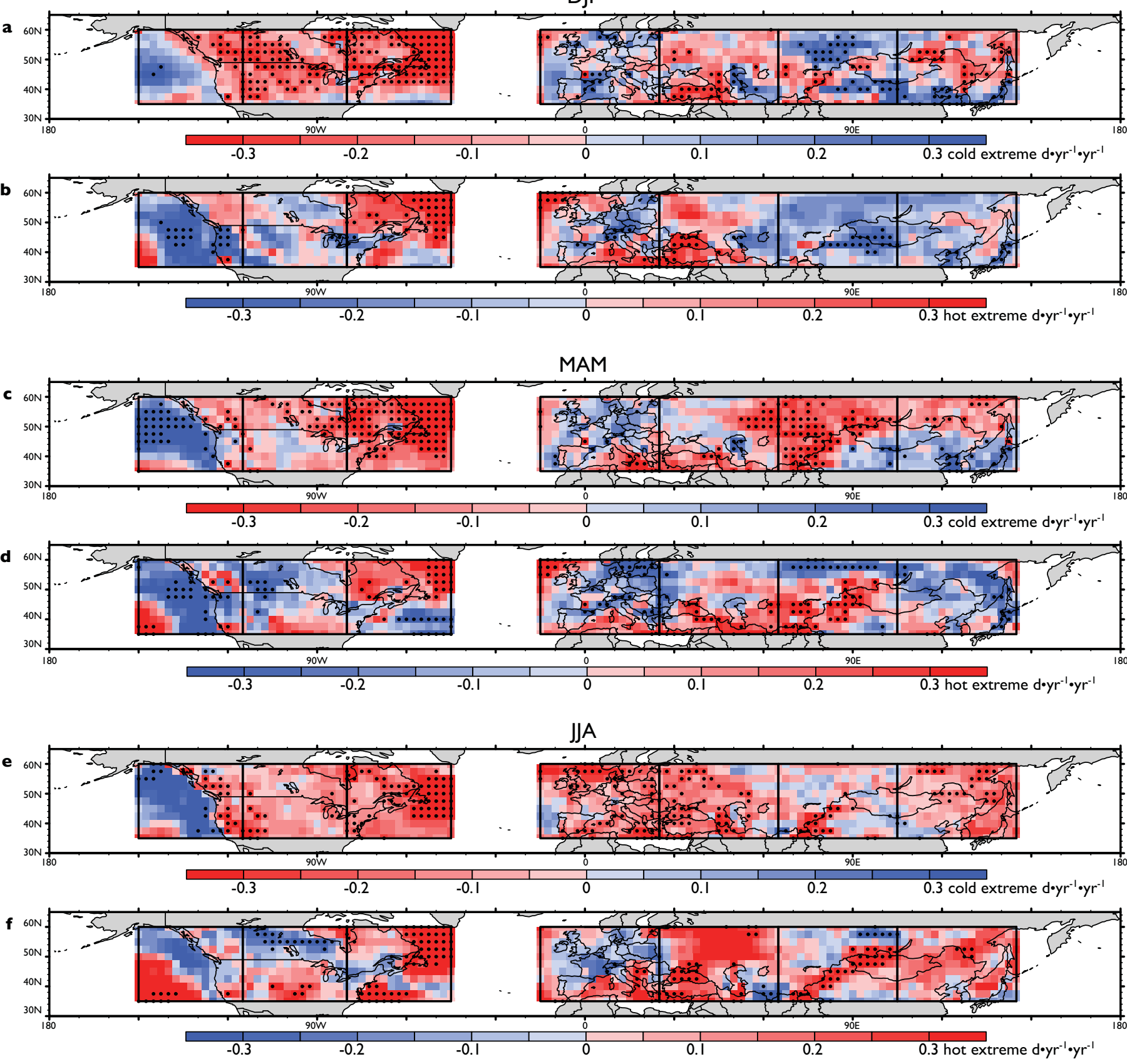

SON

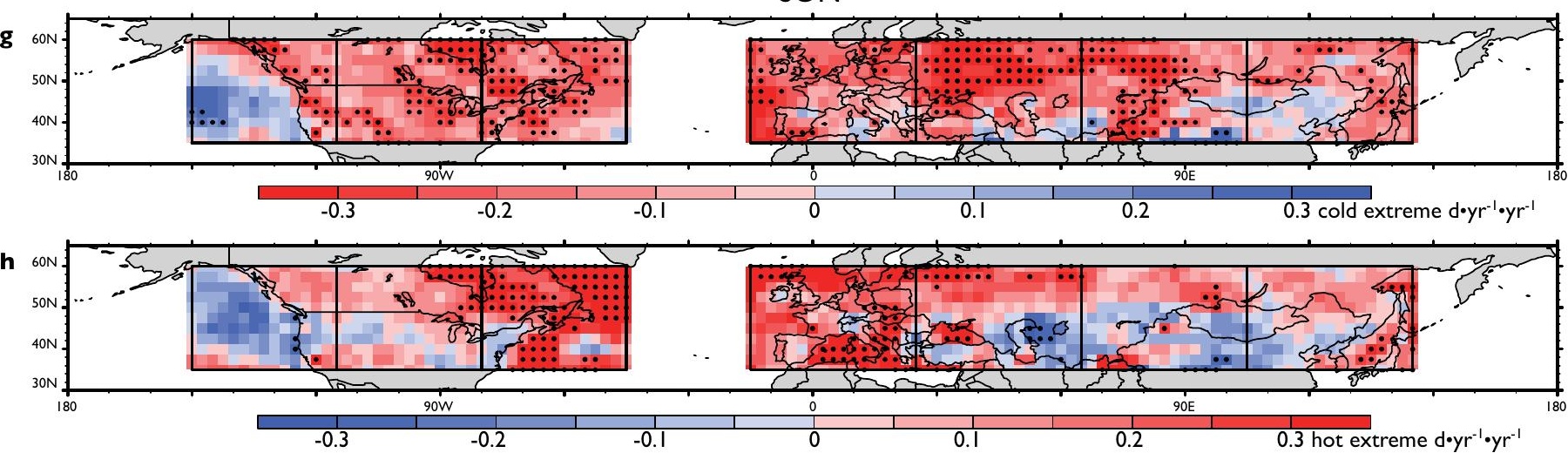




\begin{tabular}{|c|c|c|c|c|c|c|c|}
\hline \multicolumn{8}{|c|}{ Statistically Significant Trends $(p<0.05)$} \\
\hline & \multirow[b]{2}{*}{$\mathbf{n}$} & \multicolumn{3}{|c|}{ sat-era } & \multicolumn{3}{|c|}{ ice-era } \\
\hline & & OCC & per & dur & Occ & per & dur \\
\hline \multicolumn{8}{|c|}{ Linear Regression Analysis } \\
\hline NCAR/NCEP-RI & 112 & 17 & 6 & 7 & 15 & 10 & 9 \\
\hline NCEP-DOE-R2 & 112 & 16 & 5 & 7 & 13 & 8 & 9 \\
\hline ERA-Interim & 112 & 16 & 6 & 10 & 14 & 9 & 9 \\
\hline \multicolumn{8}{|c|}{ Box-Cox Transformation Analysis } \\
\hline NCAR/NCEP-RI & 112 & 15 & 9 & 8 & 16 & 9 & 10 \\
\hline NCEP-DOE-R2 & 112 & 15 & 6 & 7 & 16 & 10 & 10 \\
\hline ERA-Interim & 112 & 16 & 8 & 12 & 14 & 9 & II \\
\hline
\end{tabular}

\begin{tabular}{|c|c|c|c|c|c|c|c|}
\hline \multicolumn{8}{|c|}{ Anderson Darling Test for Normality } \\
\hline & \multirow[b]{2}{*}{$\mathbf{n}$} & \multicolumn{3}{|c|}{ sat-era } & \multicolumn{3}{|c|}{ ice-era } \\
\hline & & occ & per & dur & Occ & per & dur \\
\hline \multicolumn{8}{|c|}{ Original Data } \\
\hline NCAR/NCEP-R I & 112 & 103 & 47 & 56 & 102 & 76 & 68 \\
\hline NCEP-DOE-R2 & 112 & 102 & 57 & 58 & 102 & 74 & 70 \\
\hline ERA-Interim & 112 & 99 & 62 & 49 & 105 & 76 & 65 \\
\hline \multicolumn{8}{|c|}{$\begin{array}{c}\text { Original Data with FWER Multiple Hypothesis Testing } \\
\end{array}$} \\
\hline NCAR/NCEP-R I & 112 & 112 & 102 & 102 & 112 & 103 & 107 \\
\hline NCEP-DOE-R2 & 112 & 112 & 100 & 103 & 112 & 107 & 109 \\
\hline ERA-Interim & 112 & 112 & 100 & 102 & 112 & 104 & 106 \\
\hline \multicolumn{8}{|c|}{ Box-Cox Transformed Data } \\
\hline NCAR/NCEP-R I & 112 & 110 & 109 & 110 & 109 & 110 & 112 \\
\hline NCEP-DOE-R2 & 112 & $11 \mathrm{II}$ & 110 & 110 & 110 & $1 \mathrm{III}$ & 112 \\
\hline ERA-Interim & 112 & 110 & III & 109 & III & 109 & 108 \\
\hline \multicolumn{8}{|c|}{ Box-Cox Transformed Data with FWER Multiple Hypothesis Testing } \\
\hline NCAR/NCEP-R I & 112 & 112 & 112 & 112 & 112 & 112 & 112 \\
\hline NCEP-DOE-R2 & 112 & 112 & 112 & 112 & 112 & 112 & 112 \\
\hline ERA-Interim & 112 & 112 & 112 & 112 & 112 & 112 & 112 \\
\hline
\end{tabular}

\begin{tabular}{|c|c|c|c|c|c|c|c|}
\hline \multicolumn{8}{|c|}{ Multiple Hypothesis Testing } \\
\hline \multirow{2}{*}{$\begin{array}{l}\text { Control } \\
\text { Type }\end{array}$} & \multirow[b]{2}{*}{ Level } & \multicolumn{3}{|c|}{ sat-era } & \multicolumn{3}{|c|}{ ice-era } \\
\hline & & occ & per & dur & occ & per & dur \\
\hline \multicolumn{8}{|c|}{ NCAR/NCEP-R I } \\
\hline \multirow{2}{*}{ FWER } & $5 \%$ & 2 & 0 & 0 & 0 & $\mathrm{I}$ & 0 \\
\hline & $10 \%$ & 2 & 0 & $\mathrm{I}$ & $\mathrm{I}$ & $\mathrm{I}$ & 0 \\
\hline \multirow{2}{*}{ K-FWER } & $5 \%$ & 4 & 1 & 2 & I & 2 & 0 \\
\hline & $10 \%$ & 7 & 2 & 3 & 4 & 2 & 2 \\
\hline \multirow{2}{*}{ FDR } & $5 \%$ & 0 & 0 & 0 & 0 & 0 & 0 \\
\hline & $10 \%$ & 2 & 0 & 0 & 0 & 0 & 0 \\
\hline \multicolumn{8}{|c|}{ NCEP-DOE-R2 } \\
\hline \multirow{2}{*}{ FWER } & $5 \%$ & 2 & 0 & 0 & 0 & $\mathrm{I}$ & 0 \\
\hline & $10 \%$ & 2 & 0 & $\mathrm{I}$ & 0 & $\mathrm{I}$ & 0 \\
\hline \multirow{2}{*}{ K-FWER } & $5 \%$ & 2 & 1 & 2 & 2 & 2 & 0 \\
\hline & $10 \%$ & 5 & 2 & 4 & 5 & 3 & 0 \\
\hline \multirow{2}{*}{ FDR } & $5 \%$ & 0 & 0 & 0 & 0 & 0 & 0 \\
\hline & $10 \%$ & 2 & 0 & 0 & 0 & 0 & 0 \\
\hline \multicolumn{8}{|c|}{ ERA-Interim } \\
\hline \multirow{2}{*}{ FWER } & $5 \%$ & 0 & 0 & 1 & 0 & 0 & 0 \\
\hline & $10 \%$ & 1 & 0 & $\mathrm{I}$ & 0 & 0 & 0 \\
\hline \multirow{2}{*}{ K-FWER } & $5 \%$ & 3 & 2 & 2 & 2 & 0 & 1 \\
\hline & $10 \%$ & 7 & 2 & 2 & 5 & 3 & 3 \\
\hline \multirow{2}{*}{ FDR } & $5 \%$ & 0 & 0 & 0 & 0 & 0 & 0 \\
\hline & $10 \%$ & 0 & 0 & 0 & 0 & 0 & 0 \\
\hline
\end{tabular}


